\title{
Comparison of the properties of two fossil groups of galaxies with the normal group NGC 6034 based on multiband imaging and optical spectroscopy ${ }^{\star}$
}

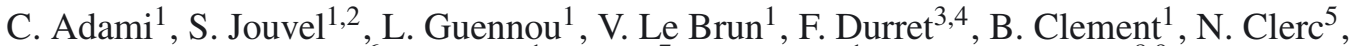 \\ S. Comerón ${ }^{6}$, O. Ilbert ${ }^{1}$, Y. Linn $^{7}$, D. Russeil ${ }^{1}$, and U. Seemann ${ }^{8,9}$ \\ ${ }^{1}$ LAM, OAMP, Université Aix-Marseille \& CNRS, Pôle de l'Étoile, Site de Château Gombert, 38 rue Frédéric Joliot-Curie, \\ 13388 Marseille 13 Cedex, France \\ e-mail: christophe.adami@oamp.fr \\ 2 UCL, Department of Physics \& Astronomy, Gower Place, London WC1E 6BT, UK \\ 3 UPMC Université Paris 06, UMR 7095, Institut d'Astrophysique de Paris, 98bis Bd Arago, 75014 Paris, France \\ ${ }^{4}$ CNRS, UMR 7095, Institut d'Astrophysique de Paris, 75014 Paris, France \\ ${ }^{5}$ Laboratoire AIM, CEA/DSM/IRFU/Sap, CEA-Saclay, 91191 Gif-sur-Yvette Cedex, France \\ ${ }^{6}$ Korea Astronomy and Space Science Institute, 776, Daedeokdae-ro, Yuseong-gu, 305-348 Daejeon, Republic of Korea \\ 7 Centre de Recherche Astrophysique de Lyon, Université de Lyon, Université Lyon 1, Observatoire de Lyon, \\ École Normale Supérieure de Lyon, CNRS, France \\ 8 Institut für Astrophysik, Georg-August Universität Göttingen, Friedrich-Hund-Platz 1, 37077 Göttingen, Germany \\ 9 European Southern Observatory, Karl-Schwarzschild-Str. 2, 85748 Garching, Germany
}

Received 7 September 2011 / Accepted 30 January 2012

\begin{abstract}
Context. Fossil groups are dominated by a bright galaxy, and their luminosity functions show an absence within half the virial radius of galaxies brighter than the central galaxy magnitude +2 . They are nevertheless massive with an extended X-ray halo. The formation and evolution of these structures is still widely debated.

Aims. To better understand the origin of these structures, it is crucial to study their faint galaxy population, as well as their large-scale environment, to determine in particular whether they are isolated or not.

Methods. We collected multiband imaging and spectroscopy for two fossil groups (RX J1119.7+2126 and 1RXS J235814.4+150524) and one normal group (associated with NGC 6034). We computed photometric redshifts in the central zones of each group, combining previous data with the SDSS five-band data. For each group we investigated the red sequence (RS) of the color-magnitude relation and computed the luminosity functions, stellar population ages and distributions of the group members. Spectroscopy allowed us to investigate the large-scale surroundings of these groups and the substructure levels in 1RXS J235814.4+150524 and NGC 6034.

Results. The large-scale environment of 1RXS J235814.4+150524 is poor, though its galaxy density map shows a clear signature of the surrounding cosmic web. RX J1119.7+2126 appears to be very isolated, while the cosmic environment of NGC 6034 is very rich. At the group scale, 1RXS J235814.4+150524 shows no substructure. Galaxies with recent stellar populations seem preferentially located in the group outskirts. A red sequence is discernable for all three groups in a color-magnitude diagram. The luminosity functions based on photometric redshift selection and on statistical background subtraction have comparable shapes, and agree with the few points obtained from spectroscopic redshifts. These luminosity functions show the expected dip between first and second brightest galaxies for the fossil groups only. Their shape is also regular and relatively flat at faint magnitudes down to the completeness level for RX J1119.7+2126 and NGC 6034, while there is a clear lack of faint galaxies for 1RXS J235814.4+150524. The faint parts of the luminosity functions appear dominantly populated by late-type galaxies.

Conclusions. RX J1119.7+2126 is definitely classified as a fossil group; 1RXS J235814.4+150524 also has properties very close to those of a fossil group, while we confirm that NGC 6034 is a normal group.
\end{abstract}

Key words. galaxies: groups: individual: NGC 6034

\section{Introduction}

The galaxy population of a fossil group is dominated by a giant elliptical (D) galaxy and includes in addition only galaxies at least two magnitudes fainter than $\mathrm{D}$ following the definition of Jones et al. (2003; see also Proctor et al. 2011, for a less stringent definition). These structures have been discovered only quite recently in the late $90 \mathrm{~s}$, concomitantly with the arrival of large-scale X-ray surveys (see e.g. Adami et al. 2011, for a recent

\footnotetext{
* Appendix A is available in electronic form at http://www. aanda.org
}

catalogue of X-ray structures including a group of galaxies very close to the fossil group status). This is because in the optical only, fossil group X-ray halos are not a priori detectable. The galaxy population of fossil groups is quite different from normal galaxy groups of similar mass, at least as far as the bright end of the galaxy luminosity function is concerned (because of the lack of galaxies in the first two magnitude bins of the luminosity functions) and the origin of these structures is still widely debated. They could result from originally truncated galaxy luminosity functions (Mulchaey \& Zabludoff 1999), or be galaxy structures that have been depopulated in terms of galaxies by 
merging processes (e.g. Cypriano et al. 2006). We also refer the reader to the recent paper by Proctor et al. (2011), which could lead to a new vision of the fossil systems formation and evolution.

To better understand the origin of these fossil structures, it is therefore crucial to study their faint galaxy populations. This is a demanding task because even for nearby objects the galaxies considered are often fainter than $\mathrm{Rc} \sim 20$. This requires large telescopes to achieve spectroscopy (e.g. Lopes de Oliveira et al. 2010, and references therein), though deep imaging can be performed with smaller telescopes (e.g. Adami et al. 2007b). It is also important to study the large-scale environment of these structures to determine whether they are isolated or not. This is also a difficult task because it requires spectroscopic samples of bright galaxies on typical scales of $\sim 100 \mathrm{deg}^{2}$. The very low surface density of these targets makes the large multi-spectrographs quite inefficient and we can use instead single-slit instruments on small telescopes to complete the bright spectroscopic catalogs already available, such as the Sloan Digitalized Sky Survey (SDSS).

We chose a triple approach, using small telescopes ( $2 \mathrm{~m}$ class) to obtain very large field spectroscopic samples of bright galaxies in the group surroundings, and direct, relatively deep imaging of the groups themselves (typically in one virial radius), and larger telescopes (4 $\mathrm{m}$ class) to obtain deep spectroscopy of faint group galaxy members. Our targets are two fossil groups (RX J1119.7+2126 and 1RXS J235814.4+150524) and one normal group (associated with NGC 6034, which we will call in the following NGC 6034 for simplicity). These data will allow us to compare the behaviors of these two different classes of structures in a homogeneous way.

Section 2 describes our data sets. Section 3 deals with the nature of the considered groups. Section 4 describes the large-scale environment of 1RXS J235814.4+150524. Section 5 gives details on the small-scale behavior of the groups (including colormagnitude relations and galaxy luminosity functions). Finally Sect. 6 is the summary.

Throughout the paper we assume $H_{0}=71 \mathrm{~km} \mathrm{~s}^{-1} \mathrm{Mpc}^{-1}$, $\Omega_{\mathrm{m}}=0.27$, and $\Omega_{\Lambda}=0.73$ (Dunkley et al. 2009). All magnitudes are given in the AB system.

\section{Sample, observations, and data characteristics}

We selected two structures among the known fossil groups (FG hereafter). We already dedicated an article to the first one (Adami et al. 2007b, on RX J1119.7+2126.7) and we present additional extensive imaging in the present work. The second group (1RXS J235814.4+150524) was selected to be one of the most distant known FGs $(z \sim 0.17$, Santos et al. 2007, but see also the $z \sim 0.6$ structure of Ulmer et al. 2005, close to the fossil group status). The third selected target is the group of galaxies associated with NGC 6034. It was originally presented as a FG by Yoshioka et al. (2004), but Lopes de Oliveira et al. (2010) showed that this group was in fact a normal group of galaxies, and we confirm this in the present work. This structure is very nearby, so we can sample its galaxy populations down to relatively deep absolute magnitudes even with small telescopes. These three groups are sampled by SDSS photometry and spectroscopic redshifts. We give their main characteristics in Table 1.

The main goals of the present work are to study their faint galaxy populations through spectroscopy and photometric redshifts and to sample the large-scale environment of these groups. We therefore collected PI observations completed by archive data as described in the following.

\subsection{Spectroscopic data}

We first compiled/reduced public Nasa Extragalactic Database (NED) and TNG (Telescopio Nazionale Galileo) spectroscopic data in the regions defined below for the three groups of the sample, and then we performed additional spectroscopy with various telescopes.

\subsubsection{Spectroscopy of bright galaxies}

As already mentioned, the isolation level of a fossil group from the cosmic web is a key element to constrain its origin. We have shown in Adami et al. (2007b) that given the field luminosity function at low redshift, the probability is low to have a galaxy brighter than the dominant fossil group galaxy in the surrounding cosmic web portions unless a massive galaxy structure is present in the region. In other words, if there is no galaxy brighter than the fossil group dominant galaxy in a given cosmological volume, the fossil group is likely to be the dominant structure of this volume. Since fossil groups are relatively minor structures, if they are the dominant structures of a given volume, this volume is also likely to be relatively poorly populated. This kind of study was already conducted by Adami et al. (2007b) for RX J1119.7+2126 and for NGC 6034 by Lopes de Oliveira (2010).

In the present work we searched for galaxies brighter than the dominant galaxy of 1RXS J235814.4+150524 $\left(r^{\prime}=16.3\right)$ in a $5 \times 5 \mathrm{deg}^{2}$ area around the group. This roughly corresponds to a $50 \times 50 \mathrm{Mpc}^{2}$ area, which agrees well with the maximum size of the known voids (Hoyle \& Vogeley 2004). To perform this search, we selected all objects classified as galaxies in the quoted area from the SDSS that are brighter than $r^{\prime}=16.3$, and do not have a known spectroscopic redshift. We visually inspected all selected objects to remove obvious saturated stars and finally had a list of 18 galaxies. Given the magnitudes of these objects, we used $2 \mathrm{~m}$ class telescopes to observe them spectroscopically. Specifically, we made observations with the CARELEC instrument mounted on the $1.93 \mathrm{~m}$ telescope at OHP in 2007 and 2008, and with the Boller \& Chivens and AFOSC instruments mounted on the $1.22 \mathrm{~m}$ and $1.8 \mathrm{~m}$ telescopes of the Asiago observatory in 2007. We successfully measured redshifts for 16 of the 18 targets and not for all of them because of time limitations. The exposure times and new redshifts acquired are listed in Table A.2, and we show an example of these 16 spectra in the appendix (see Fig. A.1). Data reductions were made with the MIDAS-based tool that was already described in Adami et al. (1998). Redshifts were measured with the EZ tool (Garilli et al. 2010) and dedicated MIDAS routines.

\subsubsection{Spectroscopy of faint galaxies}

When considering fossil groups, the two magnitude gap between the brightest and the second brightest galaxy makes any spectroscopic follow up of the faint galaxy populations difficult. $2 \mathrm{~m}$ class telescopes are therefore inefficient for this task, which is better achievable with classical multiobject spectrographs mounted on $4 \mathrm{~m}$ class telescopes. Their relatively small field of view is also well-adapted to the typical size of groups in the redshift range of interest, typically a few arcminutes on the sky.

We observed 1RXS J235814.4+150524 and RX J1119.7+ 2126 using the NTT/EFOSC2 (ID 082.A-0374) and the TNG/Dolores (2008B: CAT-9) spectrographs in 2008. The details of the observations are given in Table 2. The same table 
Table 1. Main characteristics of the three studied groups.

\begin{tabular}{|c|c|c|c|c|c|c|c|c|}
\hline Name & $\begin{array}{c}\text { RA } \\
(\mathrm{J} 2000.0) \\
\end{array}$ & $\begin{array}{c}\text { Dec } \\
(\mathrm{J} 2000.0) \\
\end{array}$ & $z$ & $\begin{array}{c}L_{\mathrm{X}, \mathrm{bol}} \\
10^{42} \mathrm{erg} / \mathrm{s} \\
\end{array}$ & $\begin{array}{l}R \text { lum. } \\
10^{11} L_{\odot}\end{array}$ & $\begin{array}{l}\text { Vel. disp. } \\
\mathrm{km} \mathrm{s}^{-1}\end{array}$ & $\begin{array}{c}\text { Virial rad. } \\
\text { Mpc }\end{array}$ & $\begin{array}{c}\text { Radial sampling } \\
\% \text { virial rad. }\end{array}$ \\
\hline RX J1119.7+2126 & $11 \mathrm{~h} 19 \mathrm{~min} 43.7 \mathrm{~s}$ & $+21 \operatorname{deg} 26^{\prime} 50^{\prime \prime}$ & 0.061 & 0.84 & 0.8 & 294 & 0.72 & $56 \%$ \\
\hline 1RXS J235814.4+150524 & $23 \mathrm{~h} 58 \mathrm{~min} 14.4 \mathrm{~s}$ & $+15 \operatorname{deg} 05^{\prime} 24^{\prime \prime}$ & 0.178 & 14.02 & 3.2 & $254 / 578$ & $0.58 / 1.31$ & $\geq 76 \%$ \\
\hline NGC 6034 & $16 \mathrm{~h} 03 \mathrm{~min} 32.1 \mathrm{~s}$ & $+17 \operatorname{deg} 11^{\prime} 55^{\prime \prime}$ & 0.034 & 13.88 & 3.1 & 297 & 0.71 & $56 \%$ \\
\hline
\end{tabular}

Notes. Name, coordinates, redshift, bolometric X-ray luminosity (in units of $10^{42} \mathrm{erg} \mathrm{s}^{-1}$ ) from Lopes de Oliveira (2010), Jones et al. (2003), and Santos et al. (2007) respectively, converted to the presently adopted cosmology and to bolometric values when needed using web PIMMS, optical R-band total luminosity, velocity dispersion, virial radius, and covered area (in percentage of the virial radius) by the imaging data.

also gives details about the data that we collected from the TNG archive facility and reduced for 1RXS J235814.4+150524 and RX J1119.7+2126.

The NTT and TNG data reductions were made with the MIDAS-based tool that was already described in Adami et al. (1998). Combining the resulting redshifts with those from the NED database, we ended up with catalogs of 43 and 32 redshifts for the 1RXS J235814.4+150524 and RX J1119.7+2126 lines of sight, respectively.

Given the sometimes limited signal-to-noise ratio of the A11 TAC-27 spectra $(S / N \leq 3)$, we were forced to smooth the data for the considered galaxies. We note that redshifts were measured with the EZ tool (Garilli et al. 2010) and dedicated MIDAS routines. To assess the quality of our redshift measurements, we also checked that multiple measurements of the same galaxies were giving similar results. Merging the galaxy sample spectroscopically measured several times in the PI spectroscopic runs (4 galaxies) and the remeasured literature galaxies with a known spectroscopic redshift (11 galaxies), we produced Fig. 1. Despite the low numbers involved, it appears that our measurements are quite secure with the exception of one galaxy that presents two clearly discrepant redshift measurements (not shown in Fig. 1). This galaxy is found at a 0.4937 redshift in the TNG data, while literature gives a value of 0.4460 . The difference arises because the literature value is placing different lines in real absorption features compared to our measurement. In the literature the presence of $\mathrm{H} \& \mathrm{~K}, \mathrm{H} \delta$, and $\mathrm{H} \beta$ was only assumed, while we are able to fit $\mathrm{H} \& \mathrm{~K}, \mathrm{H} \delta, \mathrm{G}$ band, $\mathrm{H} \gamma$, and $\mathrm{H} \beta$ in real absorption features. We are therefore assuming our value as the real redshift.

Three other galaxies (identified in Table A.3) also present slightly different redshift measurements in Fig. 1 that are on the order of 0.01 .

- For the first one $(z=0.1471$ or 0.15539$)$, the literature value is mainly based on the $\mathrm{NaD}$ line, and only offers a poor fit of the H\&K lines. Our spectrum unambiguous shows these $\mathrm{H} \& \mathrm{~K}$ lines and leads to a redshift of 0.1471 .

- The second one $(z=0.1775$ or 0.18900$)$ offers two possibilities to fit the H\&K lines. Because the NTT spectrum has a clearly poorer $S / N$, we preferred the redshift value based on the TNG spectrum $(z=0.1775)$.

- The third one ( $z=0.1745$ or 0.1793$)$ is also based on a low $S / N$ NTT spectrum and we preferred the TNG-based value of 0.1745 . The difference in redshift arises because we may fitted the $\mathrm{G}$ band and $\mathrm{H} \gamma$ instead of $\mathrm{H} \& \mathrm{~K}$ into a real double absorption line feature.

In conclusion we computed a $1 \sigma$ mean uncertainty of 0.0008 when excluding the four worst cases.

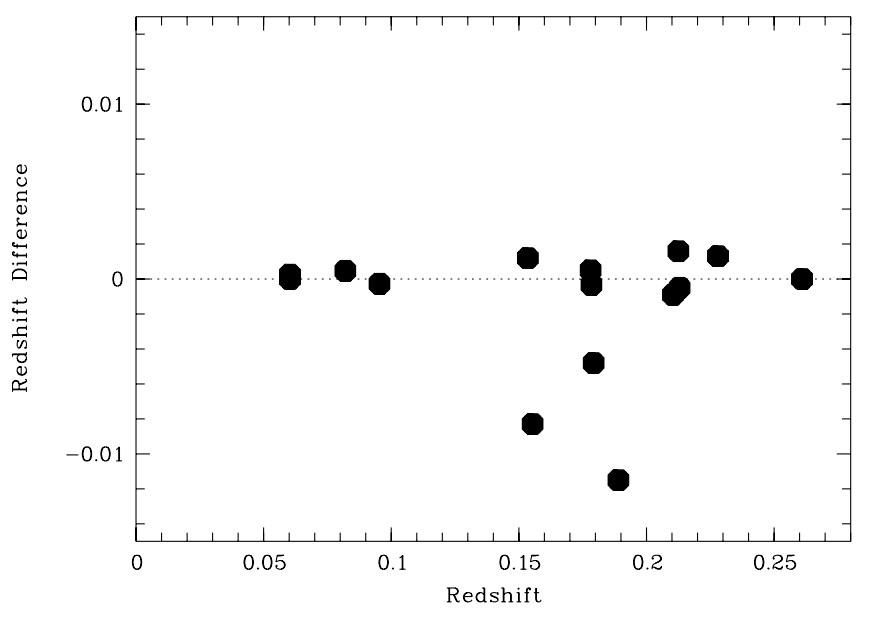

Fig. 1. Redshift difference between multiple redshift measurements as a function of redshift. The horizontal dotted line represents the zero difference.

\subsection{Optical imaging}

Two of our main goals are to constrain the luminosity function and the color-magnitude red sequence of the groups. To do this, we determined a membership criterion for faint galaxies. Our spectroscopic catalogs are far from complete at these magnitudes and we have to use photometric techniques such as photometric redshifts, color-magnitude relations or field statistical comparisons. The first step toward this goal is to obtain sufficiently deep photometric catalogs for the considered groups.

In this framework and to complement the $u^{\prime}, g^{\prime}, r^{\prime}, i^{\prime}$, and $z^{\prime}$ SDSS images, we obtained images of 1RXS J235814.4+150524 (a single field in the $R_{\mathrm{c}}$ filter) and NGC 6034 (a four field mosaic in the $B$ and $R_{\mathrm{c}}$ filters) with the $1.2 \mathrm{~m}$ OHP telescope. We also obtained multiband single-field images with the same telescope (OHP $v, B, V, g, R_{\mathrm{c}}, r, I_{\mathrm{c}}$ filters, see http://www.obs-hp.fr/ guide/camera-120/ubvri.html, Cousins 1973, 1974; Thuan \& Gunn 1976; Schneider et al. 1983) with similar depth for RX J1119.7+2126 ( $B$ and $R_{\mathrm{c}}$-band data were already presented in Adami et al. 2007b). Images were taken with the T120 $1024 \times 1024$ CCD camera and were observed under typical seeings between 2.5 and 3 arcsec. Details of the observations and of the SDSS images are given in Table 3. We also show in Fig. 2 the response functions of the selected filters.

These data map squares of $\sim 2.0 \times 2.0, \sim 0.8 \times 0.8$, and $\sim 0.8 \times$ $0.8 \mathrm{Mpc}^{2}$ for $1 \mathrm{RXS} \mathrm{J} 235814.4+150524$, RX J1119.7+2126, and NGC 6034, respectively.

We computed virial radii for the three groups from the Carlberg et al. (1997) formula, selecting as group members the galaxies in the $[0.059,0.06134]$ redshift interval for RX J1119.7+2126, in the [0.17, 0.185] and [0.177, 0.181] 
Table 2. Spectroscopic observations.

\begin{tabular}{|c|c|c|c|c|}
\hline Name & Instrument & $\begin{array}{l}\text { Exp. time } \\
\text { (s) }\end{array}$ & Grism & Allocation \\
\hline 1RXS J235814.4+150524 & ESO/NTT/EFOSC2 & 2400 & GR6 & 082.A-0374 \\
\hline RX J1119.7+2126 & TNG/Dolores & 2400 & LR-R & A18 CAT-9 \\
\hline 1RXS J235814.4+150524 & TNG/Dolores & 3600 & LR-B & A18 TAC-27 \\
\hline RX J1119.7+2126 & TNG/Dolores & $1350 / 5400 / 7200$ & MR-B & A11 TAC-27 \\
\hline
\end{tabular}

Notes. Column 1: group name; Col. 2: instrument; Col. 3: exposure time per mask; Col. 4: grism; Col. 5: allocation number. The two first lines are our own observations. The following lines are archive data.

Table 3. Imaging observations.

\begin{tabular}{|c|c|c|c|c|c|}
\hline Name & Filter & $\begin{array}{c}\text { Exposure time } \\
(\mathrm{s})\end{array}$ & Number of fields & $\begin{array}{c}\text { Seeing } \\
(\operatorname{arcsec})\end{array}$ & $\begin{array}{c}\text { ZP shift } \\
(\mathrm{mag})\end{array}$ \\
\hline NGC 6034 & $u^{\prime}$ & - & $\overline{-}$ & 1.4 & -0.15 \\
\hline NGC 6034 & $B$ & 3600 & 4 & 2.8 & 0.01 \\
\hline NGC 6034 & $g^{\prime}$ & - & - & 1.4 & -0.03 \\
\hline NGC 6034 & $R c$ & 1800 & 4 & 2.4 & 0.02 \\
\hline NGC 6034 & $r^{\prime}$ & - & - & 1.4 & -0.19 \\
\hline NGC 6034 & $i^{\prime}$ & - & & 1.1 & -0.01 \\
\hline NGC 6034 & $z^{\prime}$ & - & - & 1.3 & -0.04 \\
\hline 1RXS J235814.4+150524 & $u^{\prime}$ & - & - & 1.1 & -0.02 \\
\hline 1RXS J235814.4+150524 & $g^{\prime}$ & - & - & 1.1 & 0.06 \\
\hline 1RXS J235814.4+150524 & $R c$ & 4500 & 1 & 2.8 & -0.11 \\
\hline 1RXS J235814.4+150524 & $r^{\prime}$ & - & - & 1.1 & \\
\hline 1RXS J235814.4+150524 & $i^{\prime}$ & - & - & 1.2 & -0.22 \\
\hline 1RXS J235814.4+150524 & $z^{\prime}$ & - & - & 1.2 & -0.09 \\
\hline RX J1119.7+2126 & $u^{\prime}$ & - & - & 1.4 & 0.08 \\
\hline RX J1119.7+2126 & $v$ & 49230 & 1 & 2.7 & 0.00 \\
\hline RX J1119.7+2126 & $B$ & 10800 & 1 & 3.0 & 0.00 \\
\hline RX J1119.7+2126 & $g$ & 18000 & 1 & 2.5 & -0.04 \\
\hline RX J1119.7+2126 & $g^{\prime}$ & - & - & 1.8 & -0.15 \\
\hline RX J1119.7+2126 & $V$ & 7200 & 1 & 3.1 & 0.06 \\
\hline RX J1119.7+2126 & $R c$ & 4500 & 1 & 2.1 & 0.29 \\
\hline RX J1119.7+2126 & $r$ & 10800 & 1 & 2.3 & 0.30 \\
\hline RX J1119.7+2126 & $r^{\prime}$ & - & - & 1.7 & -0.08 \\
\hline RX J1119.7+2126 & Ic & 7200 & 1 & 3.4 & -0.05 \\
\hline RX J1119.7+2126 & $i^{\prime}$ & - & - & 1.4 & -0.18 \\
\hline RX J1119.7+2126 & $z^{\prime}$ & - & - & 1.3 & -0.11 \\
\hline
\end{tabular}

Notes. Column 1: group name, Col. 2: band, Col. 3: total duration of the summed exposures (when OHP data), Col. 4: number of different fields on the same target (when OHP data), Col. 5: seeing, Col. 6: zero point shift when computing photometric redshifts.

redshift intervals for 1 RXS J235814.4+150524, and in the [0.032, 0.037] redshift interval for NGC 6034.

- The virial radius for 1RXS J235814.4+150524 was then estimated to be 0.58 or $1.31 \mathrm{Mpc}$ (see below), so our images radius samples at least $76 \%$ of the virial area.

- The virial radius for RX J1119.7+2126 was estimated to be $0.72 \mathrm{Mpc}$, so our images radius reaches about 0.56 times the virial radius.

- The virial radius for NGC 6034 was estimated to be $0.71 \mathrm{Mpc}$, so our images radius reach about 0.56 times the virial radius.

In any case, the spatial area of interest (half the virial radius, following Jones et al. 2003) was covered for all three groups.

Data reduction was made with the MIDAS, Scamp, and Swarp (Bertin et al. 2002; Bertin 2006) packages in the same way as in Guennou et al. (2010). The resulting internal astrometric error is about 0.6 arcsec, well below the seeing of the considered images. Images in different bands were aligned at the pixel scale and object catalogs were extracted with SExtractor (Bertin $\&$ Arnouts 1996) in double image mode.

\subsubsection{Star-galaxy separation}

To distinguish between stars and galaxies, we used the plot of central surface brightness versus total magnitude shown in Fig. 3, which shows the data from the $g^{\prime}$ band SDSS images. This is an improvement on the results of Adami et al. (2007b) because OHP data were not very well adapted to perform stargalaxy separation because of poor seeing.

We were then able to efficiently distinguish galaxies from stars down to $g^{\prime}=20.4$ (see Fig. 3 for NGC 6034). We assumed that all fainter objects were galaxies, according to the results of Adami et al. (2007b).

\subsubsection{Completeness of the catalogs}

The completeness levels of the $B$ and $R_{\mathrm{c}}$ imaging data were already given in Adami et al. (2007b). We show in Figs. 4-6 the magnitude histograms of all available images. The adopted magnitude limits are also shown in the $R_{\mathrm{c}}$ band. This limit was computed considering the position of the peak of the magnitude histogram in each band, translating the value into the $R_{\mathrm{c}}$ band using 


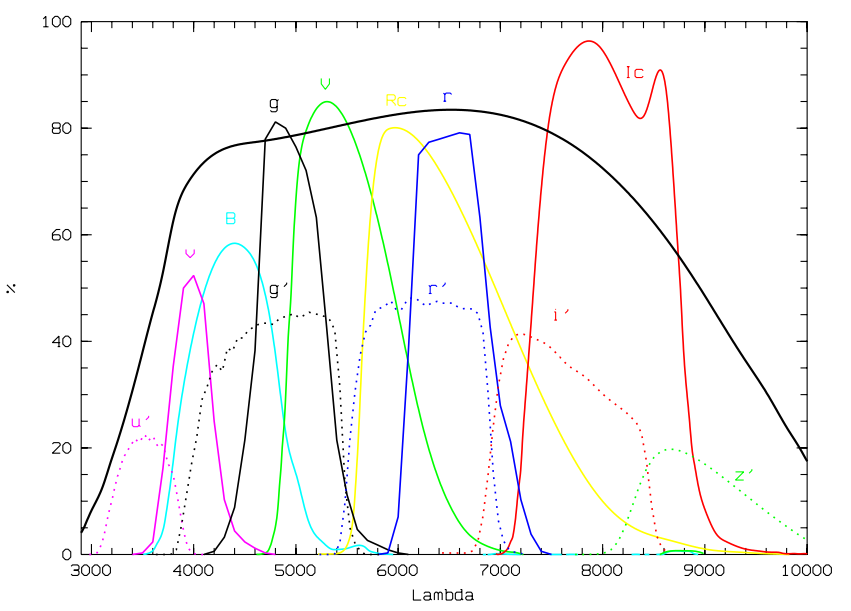

Fig. 2. Response functions of the used filters. The continuous thick black line gives the OHP T120 CCD response function. SDSS filter response functions (dotted curves) are given in arbitrary units.

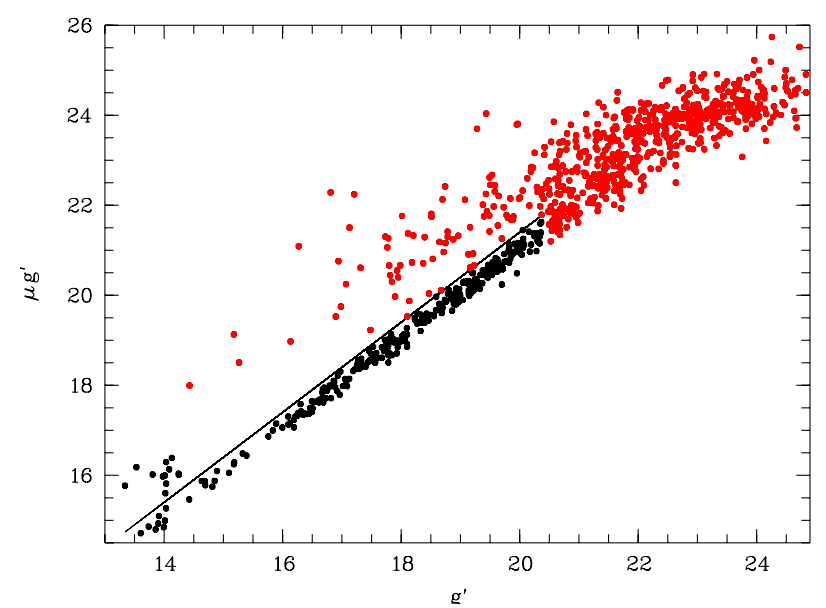

Fig. 3. Central surface brightness versus total magnitude for the NGC 6034 field in the $g^{\prime}$ band. Red dots are assumed to be galaxies and black dots are stars.

the mean color, and setting the magnitude limit to that imposed by the shallowest band (most of the time $u^{\prime}$ SDSS).

\subsubsection{Photometric redshifts}

One of the primary technical goals of the paper was to compute photometric redshifts for galaxies along the lines of sight of the considered groups of galaxies. This technique of defining a structure population with photometric redshifts is becoming common (e.g. Adami et al. 2008) but this is one of the first times to our knowledge that it is applied to fossil groups.

We could have directly used SDSS public photometric redshifts. However, these photometric redshifts are only based on a five-band survey and the number of available spectroscopic redshifts to check and adjust the photometric redshift values is quite limited in this survey for the presently considered areas, especially toward faint magnitudes. This is why we collected data both in additional photometric bands and deep spectroscopy. This gave us a six band coverage for 1RXS J235814.4+150524, seven band for NGC 6034, and twelve band for RX J1119.7+2126. We note that the OHP $R$ band data for 1RXS J235814.4+150524 were observed under variable sky conditions and calibration remained uncertain. We therefore
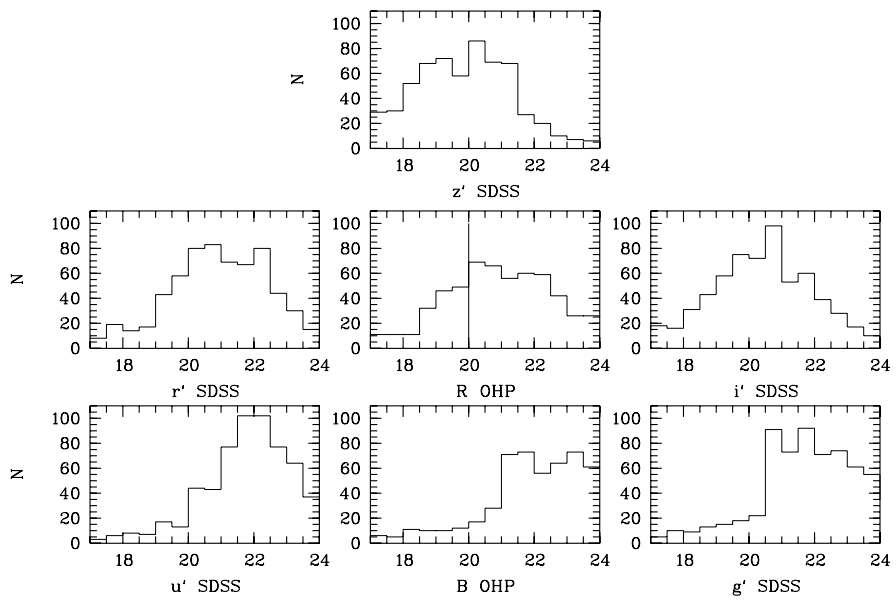

Fig. 4. Galaxy magnitude histograms from available images for NGC 6034. The adopted magnitude limit in the $R c$ band is shown.

chose to not use them when computing photometric redshifts. We then used the Le Phare code (e.g. Ilbert et al. 2006). Briefly, this photometric redshift code is able to compare observed magnitudes with predicted ones created by templates from the literature. We then selected the CE templates (see Arnouts et al. 1999) when computing simple photometric redshifts. In addition to the photometric redshifts, this gave us an estimate of the galaxy photometric type through an integer number between 1 and 66. In the present paper, the interval 1-20 was assigned to early-type galaxies (elliptical galaxy templates), 20-35 to earlyspiral galaxies (Sbc galaxy templates), and 35-66 to late-spiral galaxies (Scd and Irr galaxy templates). We also selected the Bruzual \& Charlot (2003, BC03 hereafter) templates to compute stellar population ages for galaxies with available spectroscopic redshifts. Finally, we adopted the Calzetti \& Heckman (1999) extinction law.

Le Phare was also able to estimate possible shifts in photometric band zero points, by comparing the photometric and spectroscopic redshifts used for training sets. Shifts were computed fixing photometric redshifts to the spectroscopic values and averaging the residuals in each of the bands. This technique is crucial when using photometry from observatories where the sky conditions are not always very good. We list these shifts in Table 3. They allow us to take into account internal photometry inhomogeneities between different bands.

Figure 7 shows the resulting comparisons between photometric and spectroscopic redshifts for the three groups. Given the spectroscopic catalogs we collected for our clusters (see Fig. 13 with measured galaxies down to $R_{\mathrm{c}} \sim 17.5$ for NGC 6034 and down to $R_{\mathrm{c}} \sim 21$ for the two fossil groups), Fig. 7 gives a relatively good knowledge of the quality of our photometric redshifts over nearly the entire useful magnitude range. The typical dispersions in the photometric/spectroscopic redshift relations are 0.13 for 1RXS J235814.4+150524, 0.1 for RX J1119.7+2126, and 0.06 for the NGC 6034 group when excluding the cluster redshift interval. We define in Fig. 7 the optimum photometric redshift intervals excluding as many field (fore- or back-ground) galaxies as possible and keeping as many structure galaxies as possible. The intervals are [0.13, 0.3] for 1RXS J235814.4+150524, [0., 0.095] for the NGC 6034 group, and $[0 ., 0.1]$ for RX J1119.7+2126. These intervals are shown in Fig. 7 on the vertical (photometric redshifts) and horizontal (spectroscopic redshifts) axes. On the vertical axes, they show the selection we applied to the photometric redshift catalogs. On 

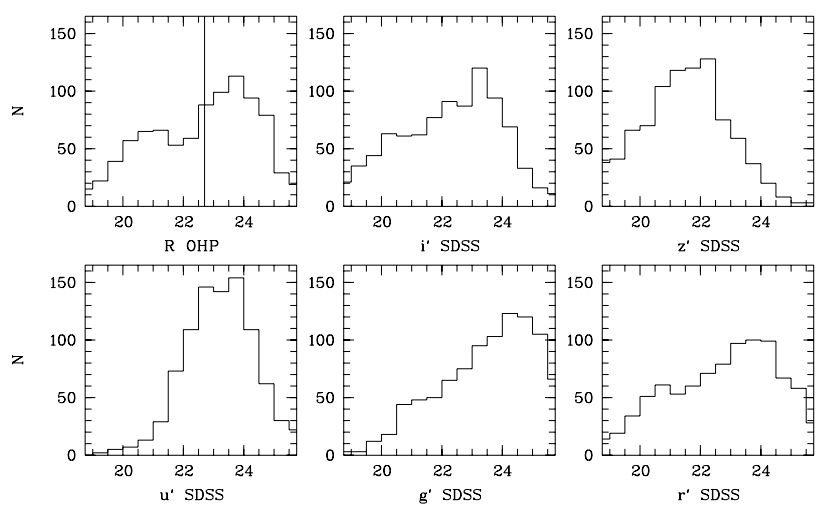

Fig. 5. Galaxy magnitude histograms from available images for 1RXS J235814.4+150524. The adopted magnitude limit in the $R_{\mathrm{c}}$ band is shown.
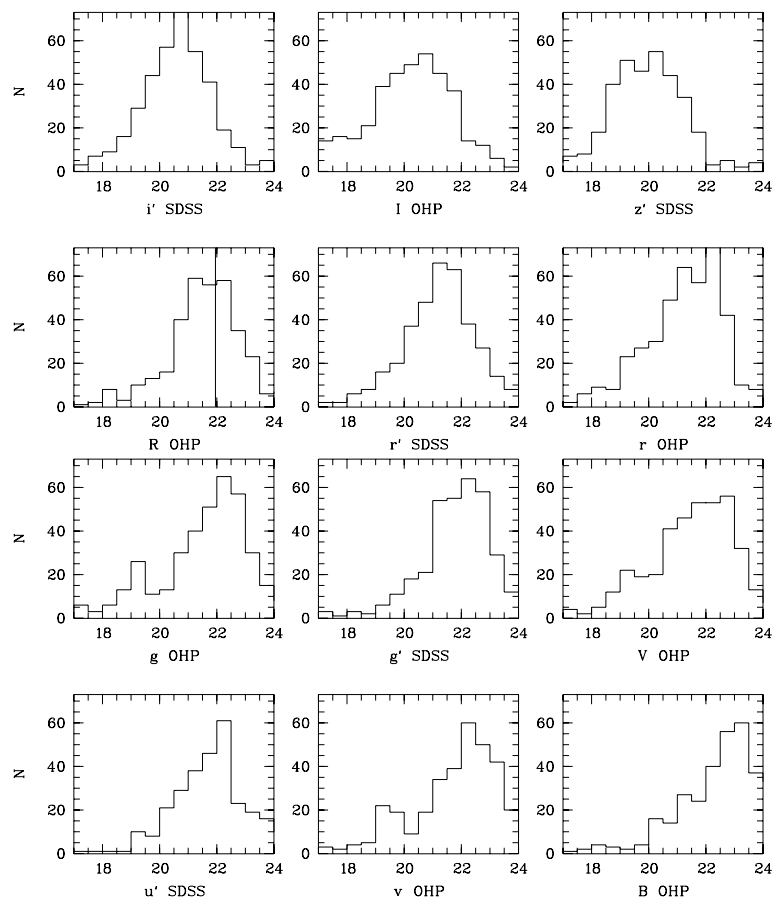

Fig. 6. Galaxy magnitude histograms from available images for RX J1119.7+2126. The adopted magnitude limit in the $R_{\mathrm{c}}$ band is shown.

the horizontal axes they illustrate that we still consider a few galaxies as group members that are fore- or back-ground objects on the basis of their spectroscopic redshift.

The spectroscopic catalogs allow us to estimate that we lose $\sim 40 \%$ of structure galaxies in this way and that we only include $\sim 10 \%$ of field galaxies in 1RXS J235814.4+150524. The percentage of lost structure members is important, but $\sim 80 \%$ of these lost galaxies are late-type objects. This means that we are able to distinguish efficiently between early-type galaxies that lie inside and outside of the $[0.13,0.3]$ redshift interval for 1RXS J235814.4+150524. This tendency to have degraded photometric redshift estimates for relatively bright cluster late-type galaxies was already shown in Guennou et al. (2010) and is probably caused by a lack of literature late-type templates adapted to high-density environments.

For the NGC 6034 group, we also lose $30 \%$ of structure galaxies and include $\sim 10 \%$ of field galaxies. Similarly to 1RXS J235814.4+150524, all lost galaxies are late-type objects, so we are able to efficiently distinguish between early-type
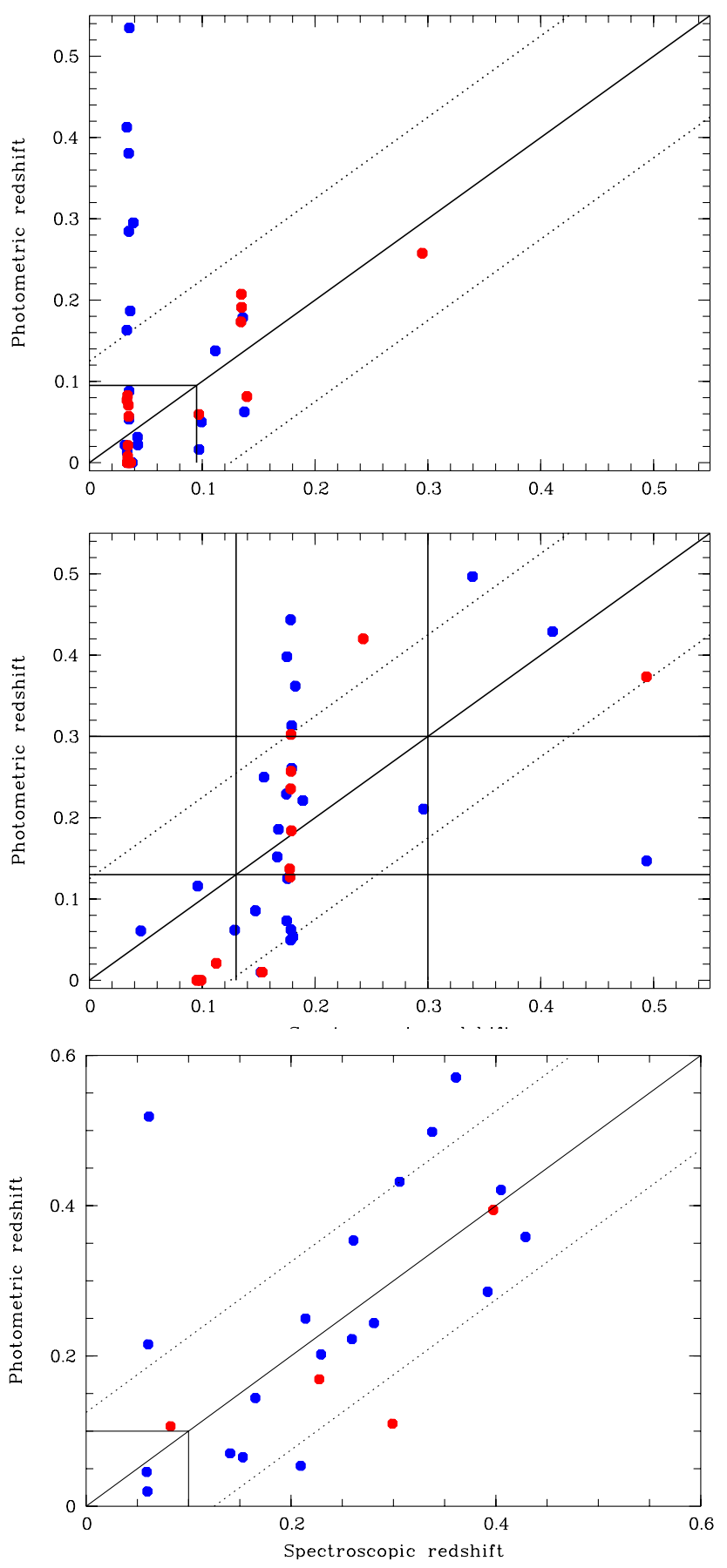

Fig. 7. Spectroscopic versus photometric redshifts for the NGC 6034 group (top), the 1RXS J235814.4+150524 fossil group (middle), and the RX J1119.7+2126 fossil group (bottom). Red dots are early-type galaxies, while blue dots are spiral galaxies (see text). The inclined lines show the perfect relation and its $\pm 0.1251 \sigma$ uncertainty. We also show the region chosen as the "structure" redshift range.

galaxies that lie inside and outside of the [0., 0.095] redshift interval for the NGC 6034 group.

It is not possible to reliably estimate the percentages of lost structure galaxies and included field galaxies for RX J1119.7+2126, because of the few spectroscopic redshifts lying inside the structure, so we chose the same limitations as for NGC 6034. This proved to give consistent results when computing luminosity functions estimated with photometric redshifts and with statistical subtraction techniques. 


\section{Nature of the studied groups}

The first question is to know if we are indeed dealing with fossil groups in our sample, since partial data can sometimes lead to wrong interpretations of the nature of a given group. For example, NGC 6034 was claimed to be a fossil group by Yoshioka et al. (2004). However, considering new optical data, Lopes de Oliveira et al. (2010) showed that this group was not a member of the fossil class.

We first confirm that the NGC 6034 galaxy is not associated with a fossil group. From available spectroscopic redshifts in the literature, two group member galaxies are less than 2 mag fainter than NGC 6034 and are located at 185 and $352 \mathrm{kpc}$ from this galaxy. Lopes de Oliveira et al. (2010) estimated the virial radius of this group to be $940 \mathrm{kpc}$. The two mentioned galaxies are therefore inside half the virial radius.

RX J1119.7+2126 was classified as a fossil group by Jones et al. (2003). The spectroscopic data of the present paper, already partly used in Adami et al. (2007b), show that besides the dominant galaxy, the second brightest galaxy member of the group and within a circle of radius $360 \mathrm{kpc}$ is $2.53 \mathrm{mag}$ fainter in the $R$ band. There is only one galaxy less than 2 mag fainter than the dominant galaxy, without spectroscopic redshift, which is potentially a group member from photometric redshift results. This galaxy is 1.94 mag fainter than the dominant galaxy and is outside half the virial radius of the group. Finally, the X-ray luminosity is high enough to classify RX J1119.7+2126 as a fossil group following Jones et al. (2003).

1RXS J235814.4+150524 is luminous enough in X-rays (Santos et al. 2007) to be potentially considered as a fossil group. The velocity dispersion of this group is at most $578 \mathrm{~km} \mathrm{~s}^{-1}$ considering our spectroscopically measured galaxies between $z=0.17$ and 0.185 (or $254 \mathrm{~km} \mathrm{~s}^{-1}$ when considering galaxies between $z=0.177$ abd $z=0.181$ ). With the virial radius definition of Carlberg et al. (1997), this corresponds to 1.31 Mpc. With the spectroscopic catalogs in hand, there are only two group member galaxies in the available field of view that are less than two magnitudes fainter than the dominant galaxy. The secondbrightest galaxy in our field of view is at $1.015 \mathrm{Mpc}$ from the dominant galaxy; it is therefore beyond half the virial radius and cannot be used to invalidate the fossil nature of the considered group following Jones et al. (2003). The third-brightest galaxy of RXJ 2359 is very close to the central galaxy and the magnitude difference between these two galaxies is $1.8 \mathrm{mag}$ in the $R_{\mathrm{c}}$ band.

From the photometric redshift catalog, there are three potential group member galaxies in the available field of view which are less than two magnitudes fainter than the dominant galaxy. The second brightest of these galaxies is not a member galaxy (this galaxy has a spectroscopic redshift outside of the group). The two next galaxies are at more than $750 \mathrm{kpc}$ from the dominant galaxy and are therefore beyond half the virial radius.

At the end, only the third brightest galaxy in the spectroscopic sample is bright and close enough to the dominant galaxy to violate the fossil group definition. However, given the difference of nearly two magnitudes, we can say that RXJ 2359 is very close to the fossil group status from the Jones et al. (2003) definition (see also Sect. 5.5 of the recent paper by Proctor et al. 2011).

\section{Large-scale environment of the fossil groups}

On the one hand, we have already shown in Adami et al. (2007b) that RX J1119.7+2126 is very isolated from the cosmic web. On the other hand, Lopes de Oliveira et al. (2010) showed that

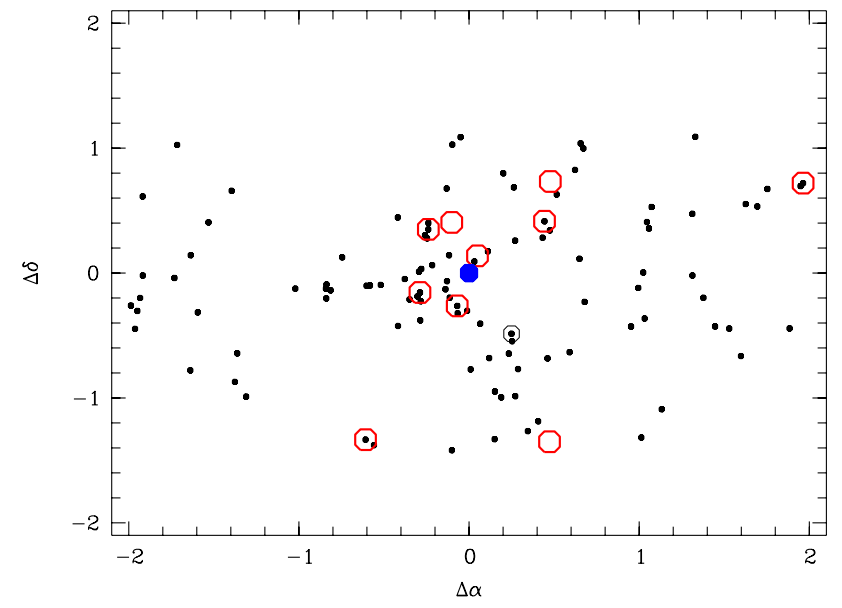

Fig. 8. Map of the large-scale environment of the 1RXS J235814.4+ 150524 fossil group (blue disk). Black dots are the known galaxies within the redshift range [0.16; 0.195]. The black circle shows the only galaxy less than two magnitudes fainter than the dominant galaxy. Red circles are the known compact galaxy structures (groups or clusters) in the same redshift range. Units of the figure are decimal degrees relative to the group center.

NGC 6034 is highly connected to the cosmic web with at least twelve clusters of galaxies at similar redshifts in a two-degree field.

Figure 8 shows the large-scale environment of the 1RXS J235814.4+150524 fossil group in a $\sim 50 \mathrm{Mpc}$ area. We first note that ten compact structures of galaxies (groups or clusters from NED) are known in this area in the redshift range [0.16;0.195]. 1RXS J235814.4+150524 is therefore much less isolated than RX J1119.7+2126. However, only one galaxy in this area is less than two magnitudes fainter than the dominant galaxy of 1RXS J235814.4+150524 (so even fainter than the considered condition to be only fainter than the dominant galaxy). As demonstrated in Adami et al. (2007b), this probably means that 1RXS J235814.4+150524 is the dominant structure in this region and that the ten compact galaxy structures detected are probably very low mass systems. 1RXS J235814.4+150524 is therefore not acting as a negligible system in its cosmological bubble, as was the case for the NGC 6034 group, which is minor compared to the giant clusters in its vicinity.

We conclude that the fossil group 1RXS J235814.4+150524 is only embedded in a relatively diffuse environment, therefore not providing many infalling galaxies.

\section{Small-scale properties of the groups}

We will now investigate the properties of the faint galaxy populations of the present group sample.

\subsection{Spatial distributions of the group members}

Lopes de Oliveira et al. (2010) showed density contours of the likely members of the NGC 6034 group in their Fig. 3. However, this plot is probably partially polluted by field galaxies. Here, we selected likely group members by considering only galaxies with photometric redshifts inside the intervals defined earlier. As stated before, this will mainly give the distribution of the early-type galaxies of the group, since we lose a considerable part of the late-type galaxies. We also note that several field galaxies will still be included in the group population because 


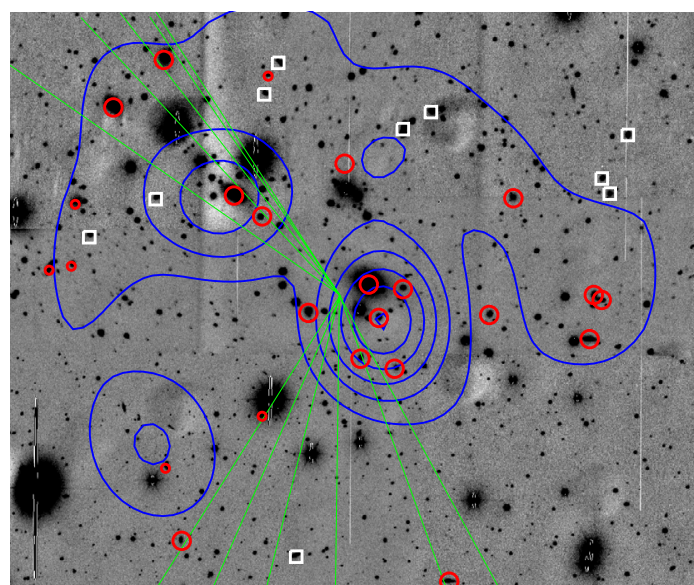

Fig. 9. Adaptative kernel galaxy density map of galaxies with photometric redshifts lower than 0.095 along the NGC 6034 line of sight (blue contours). The first contour corresponds to the mean galaxy density value over the field of view, and the last contour to the maximal value. The size of the image is $20 \times 20 \mathrm{arcmin}^{2}$. North is up and east is to the left. Red circles are galaxies with a spectroscopic redshift inside the group (small and large circles are galaxies with mean stellar population younger and older than $10^{10} \mathrm{yr}$ ). White squares are galaxies with spectroscopic redshift outside the group. Green lines represent the directions to the closest galaxy clusters (see Lopes de Oliveira et al. 2010).

photometric redshifts are not as precise as spectroscopic ones. However, following Ilbert et al. (2005), we estimated that this contribution is small for the three considered groups (less than one galaxy per half magnitude and per $\mathrm{deg}^{2}$ ). We then computed a galaxy density map from this sample with an adaptive kernel code as described in Adami et al. (2007a). The result is shown in Fig. 9. We clearly see the main part of the structure as well as a north-east extension correlated with a relatively bright galaxy (a group member) and in the direction of the Abell 2151 complex. These results are consistent with the picture drawn by Lopes de Oliveira et al. (2010) in which the NGC 6034 group is part of a larger complex that includes several clusters of galaxies.

Figure 10 shows the galaxy density map for galaxies with photometric redshifts in the $z=[0.13,0.3]$ range along the 1RXS J235814.4+150524 line of sight. Once again, we clearly detect the core of the fossil group. Density contours are less extended than for the NGC 6034 group but seem to show a south extension nicely correlated with the direction of three close compact galaxy structures. We therefore at least partially confirm the results of the previous section: 1RXS J235814.4+150524 appears to be a relatively isolated structure, but with a clear signature of the surrounding cosmic web.

We already computed a member galaxy density map for RX J1119.7+2126 in Adami et al. (2007b) based on a $\left(B-R_{\mathrm{c}}\right)$ color selection. We present here the new results based on the 12-band photometric redshift analysis (Fig. 11). The density map computed here is very similar to Fig. 6 of Adami et al. (2007b), also showing the north and east concentrations, which confirms that the immediate vicinity of the dominant galaxy is strongly depopulated in terms of galaxies.

To confirm the isolation status of the fossil groups compared to the NGC 6034 group, we also searched for recent infalling activity through substructure analysis. This is not possible for RX J1119.7+2126 because we have detected only five spectroscopic members. For 1RXS J235814.4+150524, which is not very massive and is the dominant structure of its area

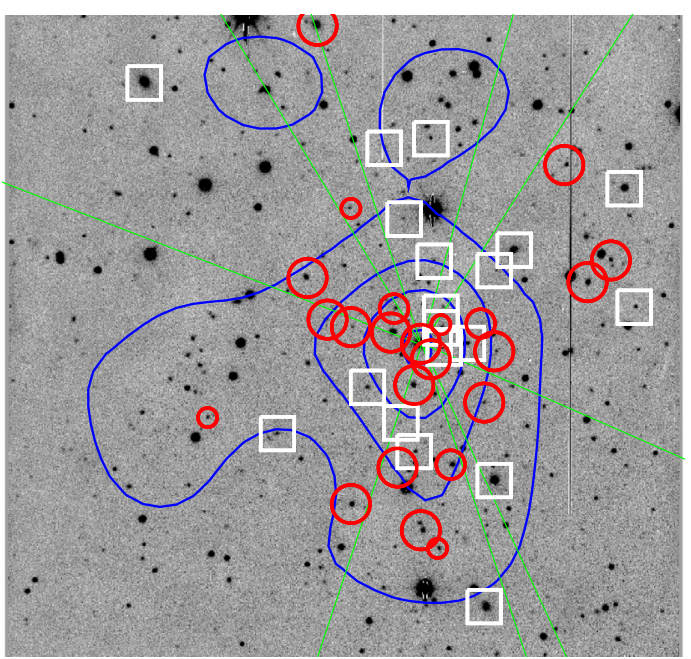

Fig. 10. Adaptative kernel galaxy density map of galaxies with photometric redshifts in the $[0.13,0.3]$ redshift interval along the 1RXS J235814.4+150524 line of sight (blue contours). The first contour corresponds to the mean galaxy density value over the field of view, and the last contour to the maximal value. The size of the image is $11.6 \times 11.6 \mathrm{arcmin}^{2}$. North is up and east is to the left. Symbols are the same as in Fig. 9. Green lines represent the direction of the closest known compact structures of galaxies from NED.

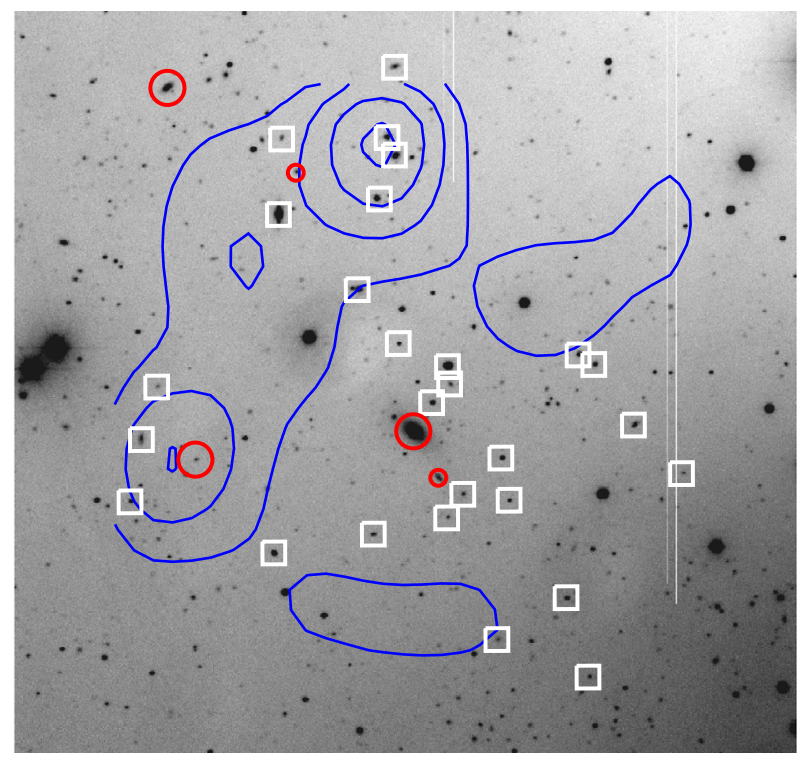

Fig. 11. Adaptative kernel galaxy density map of galaxies with photometric redshifts in the $[0 ., 0.1]$ redshift interval along the RX J1119.7+2126 line of sight (blue contours). The first contour corresponds to the mean galaxy density value over the field of view, and the last contour to the maximal value. The size of the image is $11.5 \times 11.5 \mathrm{arcmin}^{2}$. North is up and east is to the left. Symbols are the same as in Fig. 9. The fossil group main galaxy is at the image center.

(see previous section), we expect to find only minor substructuring, indicative of a weak infalling activity. We performed a Serna-Gerbal substructure search (Serna \& Gerbal 1996) on the spectroscopic catalog of 1RXS J235814.4+150524 and detected no substructure. With the available spectroscopic catalog, major substructures should be detected if present (see e.g. Adami et al. 2011). As a comparison, we detected a potential substructure in the NGC 6034 group with an estimated virial mass of $4 \times 10^{12} M_{\odot}$, typically one tenth of the mass of the parent group 
C. Adami et al.: Comparison of the properties of two fossil groups of galaxies with the normal group NGC 6034
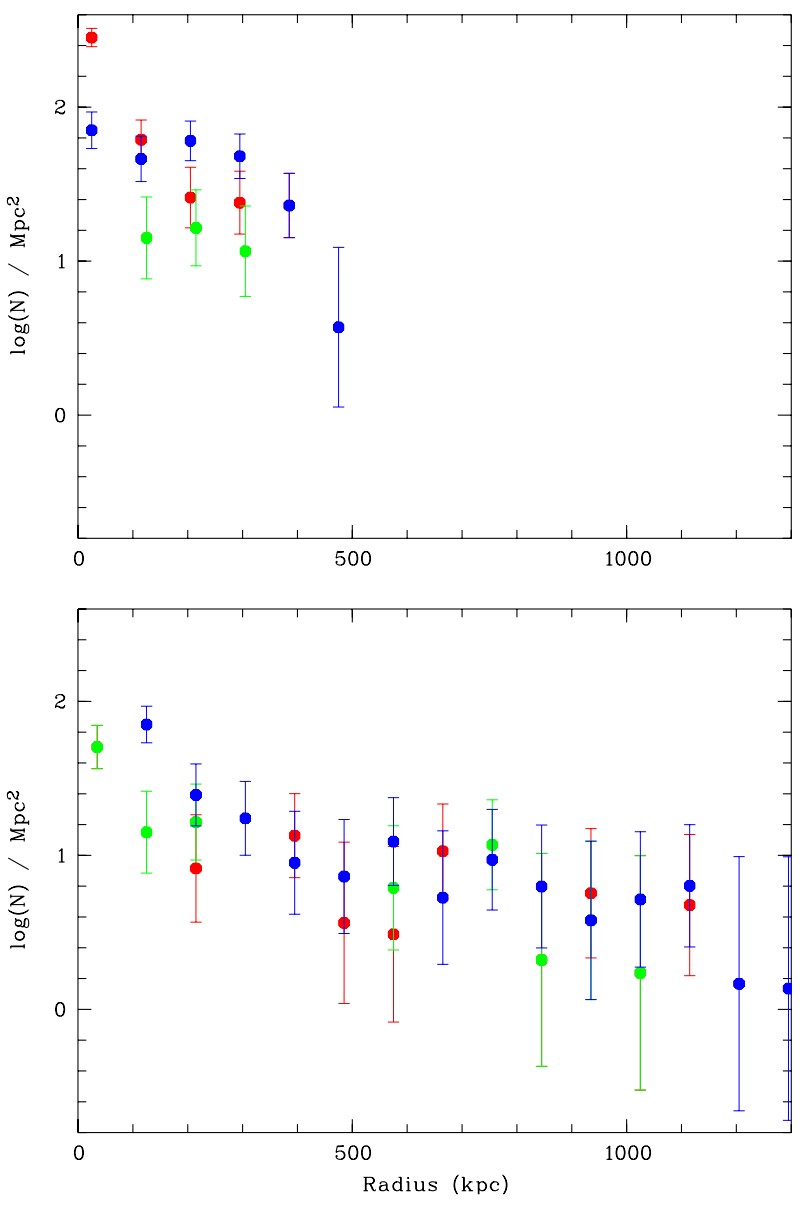

Fig. 12. Galaxy density profiles of elliptical (red dots), early spiral (green dots), and late spiral (blue dots) galaxies for NGC 6034 (top) and 1RXS J235814.4+150524 (bottom). Bin size for the two figures was $90 \mathrm{kpc}$.

itself (see Lopes de Oliveira et al. 2010). The main galaxy of this substructure is the central galaxy of the secondary peak located northeast of Figs. 9. The NGC 6034 group therefore seems to have accreted a very small group coming from the surrounding cosmic web, while 1RXS J235814.4+150524 does not show a similar behavior.

The photometric redshift computation process also produces estimates of the photometric type of the galaxies, as already stated. Keeping in mind that we are dealing with catalogs of potential structure members biased toward early-type galaxies, we computed galaxy density profiles for the considered groups as a function of galaxy type (Fig. 12). Since galaxy density contours in Figs. 9 and 10 are relatively concentrated and isotropic, we computed numbers of galaxies within annuli for these two groups, limiting the galaxy samples to a common absolute magnitude value given by the shallowest data. Only galaxies brighter than $M_{R_{\mathrm{c}}}=-16.85$ were kept.

Figure 12 shows that the NGC 6034 and 1RXS J235814.4+ 150524 groups show similar galaxy density profiles with similar density values. There is one main difference, however: while 1RXSJ235814.4+150524 basically shows a flat profile close to the center, NGC 6034 is more cuspy, mainly because of elliptical-like galaxies.

These possible tendencies are not taking into account magnitude effects, however. We therefore compute the colormagnitude relations and the luminosity functions of the three groups.

\subsection{Color-magnitude relations}

Color-magnitude relations (CMR hereafter) are very useful to sample galaxy populations, in particular since cluster early-type galaxies most of the time exhibit a red sequence (RS hereafter) that is characteristic of their evolution (e.g. Kodama \& Arimoto 1997). We already showed such RS in the CMR of RX J1119.7+2126 in Adami et al. (2007b), based on statistical arguments, and a similar RS was also detected in the NGC 6034 group by Lopes de Oliveira et al. (2010). We show in Fig. 13 the color-magnitude relations of the three groups computed with the present data. RS are discernable in the three cases, confirming that we are probably dealing with sufficiently old structures that had time to assemble an old and early-type galaxy population.

\subsection{Galaxy luminosity functions of the fossil groups}

We know that fossil groups only have a faint galaxy population (at least two magnitudes fainter than the brightest object) in addition to their brightest galaxy. The previous section considered galaxies in the whole observed area. To check whether the considered fossil groups in the present paper include galaxies in the dwarf regime or not, we now compute luminosity functions in half the virial radius. This is the area where faint galaxy populations could be the most affected.

To compute luminosity functions in the $R_{\mathrm{c}}$ band for the three groups, we first considered the photometric redshifts to define the group membership, as we did for Coma (Adami et al. 2008). Briefly, the photometric redshifts were used to remove as many field galaxies as possible (with the limitations quoted in the previous section). This was mainly efficient for earlytype galaxies. Then, we statistically subtracted the field luminosity function in the same band and in the considered volume with the Ilbert et al. (2005) estimates. We show the results in Fig. 14. On the one hand, we confirm the lack of faint galaxies in 1RXS J235814.4+150524; we may have reached the luminosity function turnover of this structure. On the other hand, the luminosity functions of the NGC 6034 and RX J1119.7+2126 groups have more regular shapes down to the completeness level, with a relatively flat slope, if we except the expected dip between the dominant and the second-brightest galaxy for RX J1119.7+2126.

Given the effects that are sometimes important for galaxy photometric type selection on the membership criterion, we also computed luminosity functions with other methods. We first used the spectroscopic catalog for 1RXS J235814.4+150524 assuming that no selection bias was present (this was not possible for RX J1119.7+2126 because of the few spectroscopic members). For a given magnitude interval (chosen to include at least ten galaxies with a spectroscopic redshift inside the galaxy structure), we computed the ratio between the group spectroscopic members and the field galaxies. We then applied this ratio to the photometric catalogs. This allowed us to put a few spectroscopic-based points in Fig. 14. These points are within the error bars of the photometric redshift-based galaxy luminosity functions. However, spectroscopy is not always deep enough to sample the faint magnitude regime. We therefore also computed luminosity functions with a pure statistical approach. We statistically estimated the back- and foreground contributions along the considered lines of sight with the comparison fields already used in Adami et al. (2007b). Since they were observed in sky regions of low extinction with the same telescope, CCD, and $R_{\mathrm{c}}$ filter, they allowed us to statistically subtract field galaxies. Results are shown in Fig. 15 for the $R c$ band. Despite the larger 

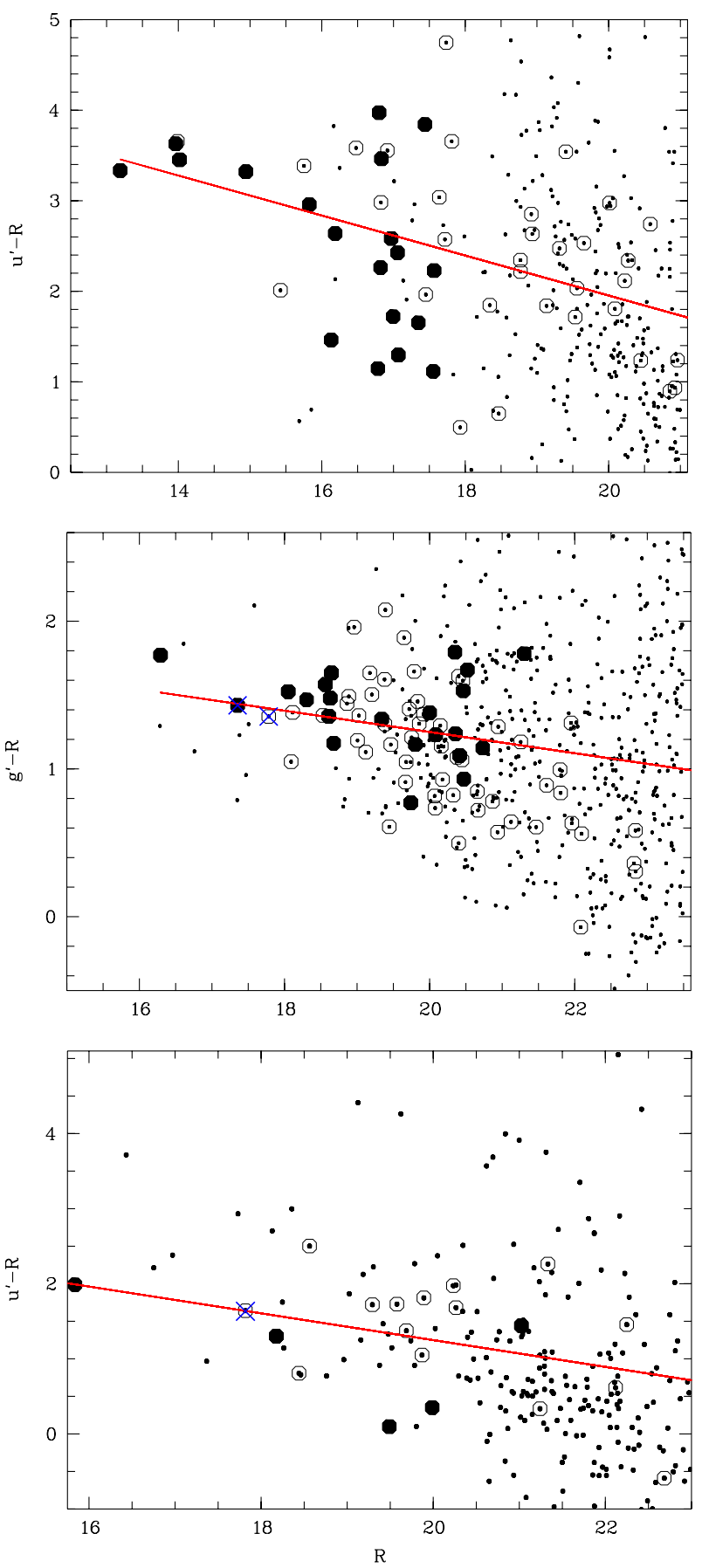

Fig. 13. Color-magnitude relations for NGC 6034 (top), 1RXS J235814.4+150524 (middle), and RX J1119.7+2126 (bottom). Dots: all galaxies detected along the lines of sight. Filled circles: galaxies spectrocopically classified as group members. Open circles: galaxies that potentially are members of the group from their photometric redshift. The blue crosses (middle and lower figures) are potential group members (from photometric or spectroscopic redshifts) less than two magnitudes fainter than the dominant group galaxy but which are more distant than half the virial radius from the group center. They are therefore no indrance to classify the group as a fossil structure. The red lines are the mean red sequences computed selecting spectroscopic group members and potential members through photometric redshifts.

error bars, results are consistent with Fig. 14 except perhaps for RX J1119.7+2126.

The computed luminosity functions also allow us to compute the total $R$-band luminosity of the three groups. Simply
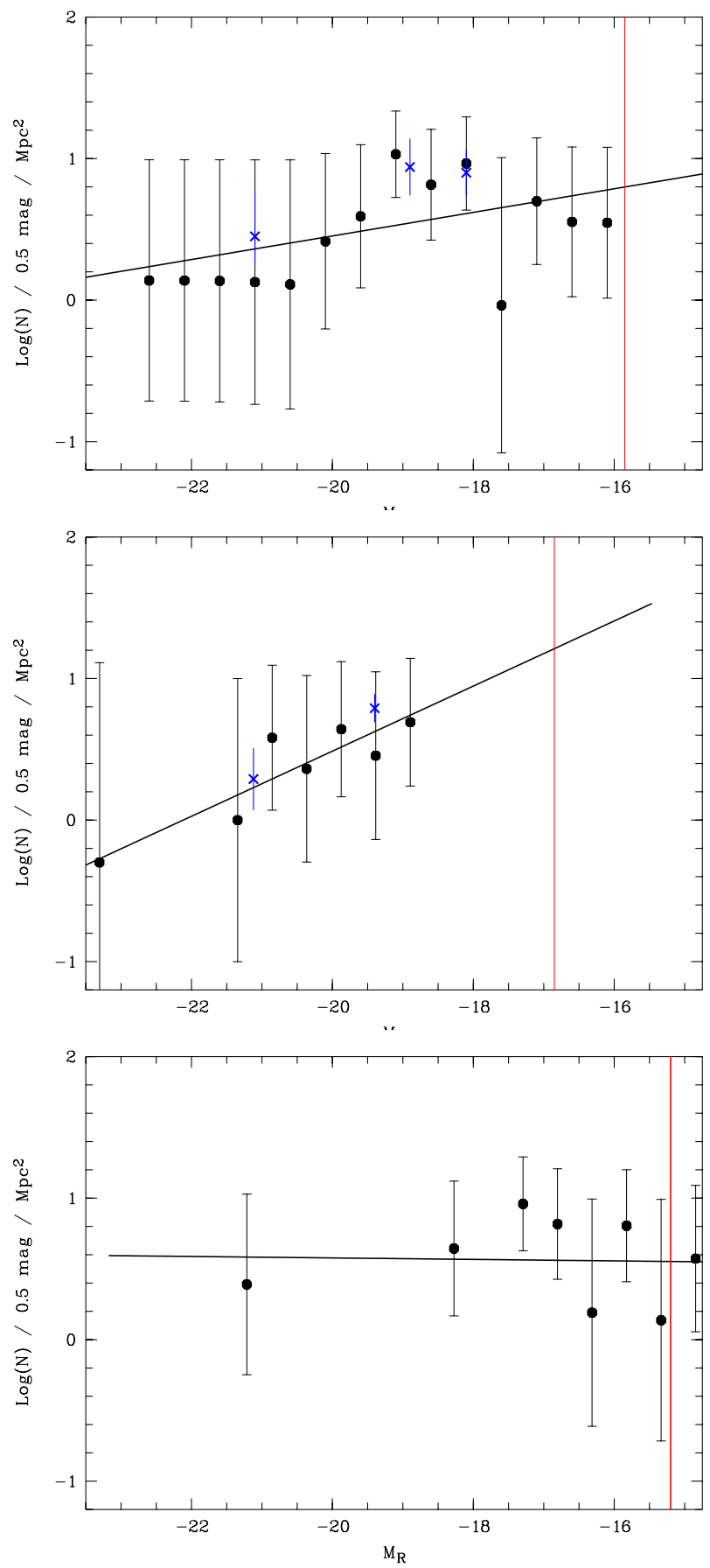

Fig. 14. Photometric redshift based $R_{\mathrm{c}}$ band galaxy luminosity functions of NGC 6034 (top), 1RXS J235814.4+150524 (middle), and RX J1119.7+2126 (bottom). The black lines are the mean luminosity functions of the $M_{R_{\mathrm{c}}}$ intervals [-22.5, -16.] (NGC 6034), [-22.5, -19.] (1RXS J235814.4+150524), and [-21.5, -15.25] (RX J1119.7+2126]. The red lines are the adopted completeness levels. The blue crosses are from the spectroscopic catalogs.

integrating the photometric redshift-based galaxy luminosity functions in the magnitude ranges considered in Fig. 14 and assuming $L_{\odot_{R}}=4.62$, we obtained the total $R$-band luminosities listed in Table 1. It is interresting to note that RX J1119.7+2126 has a very low optical luminosity which agrees well with the estimates of Jones et al. (2003). Moreover, we also see that NGC 6034 and 1RXS J235814.4+150524 have similar $R$-band (and X-ray bolometric) luminosities, even though one is a 
C. Adami et al.: Comparison of the properties of two fossil groups of galaxies with the normal group NGC 6034
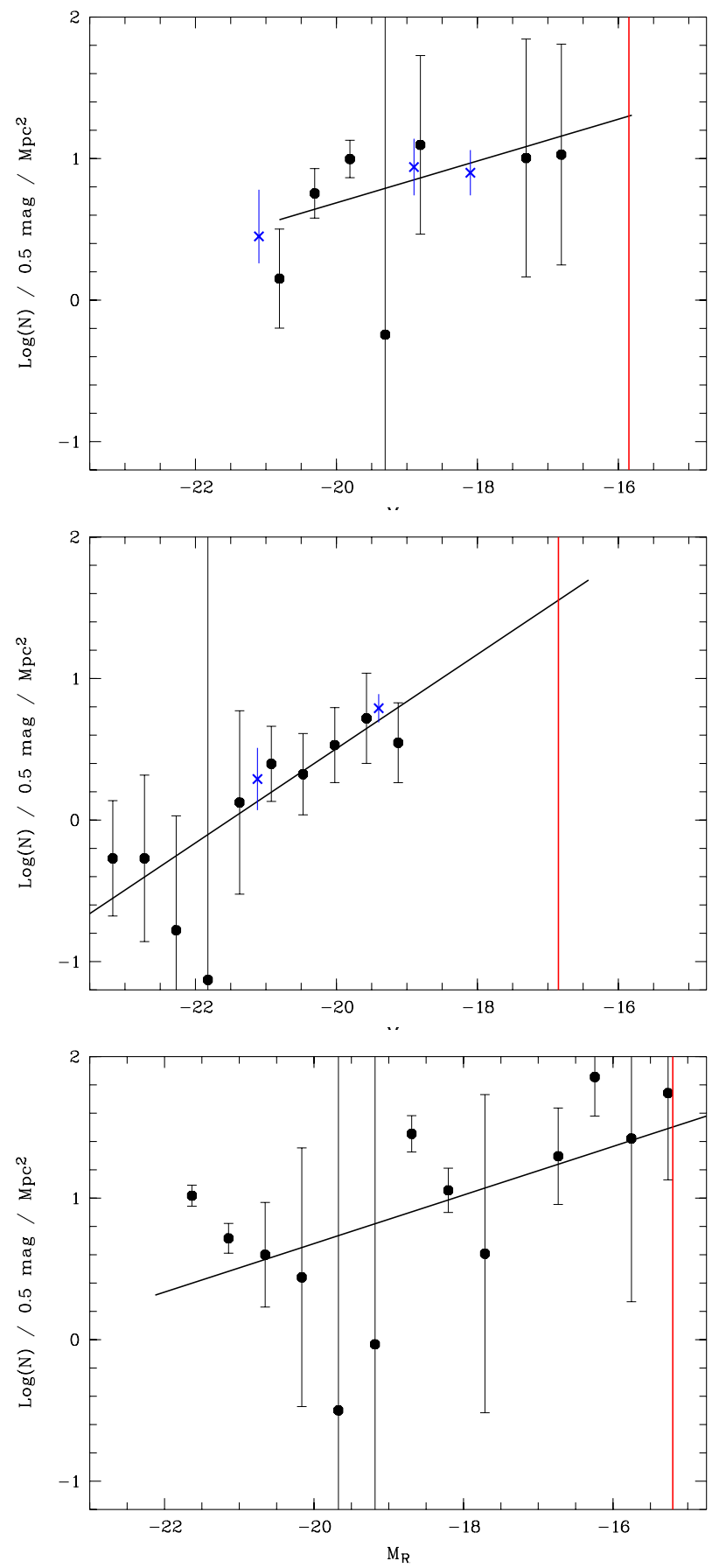

Fig. 15. Statistically-subtracted Rc-band galaxy luminosity functions of NGC 6034 (top), 1RXS J235814.4+150524 (middle), and RX J1119.7+2126 (bottom). The black lines show the mean luminosity functions in the $M_{R_{\mathrm{c}}}$ intervals $[-21,-18$.$] (NGC 6034) and [-22., -19.]$ (1RXS J235814.4+150524). The red lines are the adopted completeness levels. The blue crosses are from the spectroscopic catalogs.

normal group and the other is a fossil group. NGC 6034, however, is a group with galaxies spread over a wide range of magnitudes, as opposed to 1RXS J235814.4+150524, which shows the well known 2-mag gap after the group dominant galaxy, many relatively bright galaxies $\left(M_{R} \sim-20\right)$ after this gap, and no galaxy members fainter than $M_{R} \leq-19$.

The last question we adress in this section is the relative contribution of the different galaxy photometric types to these
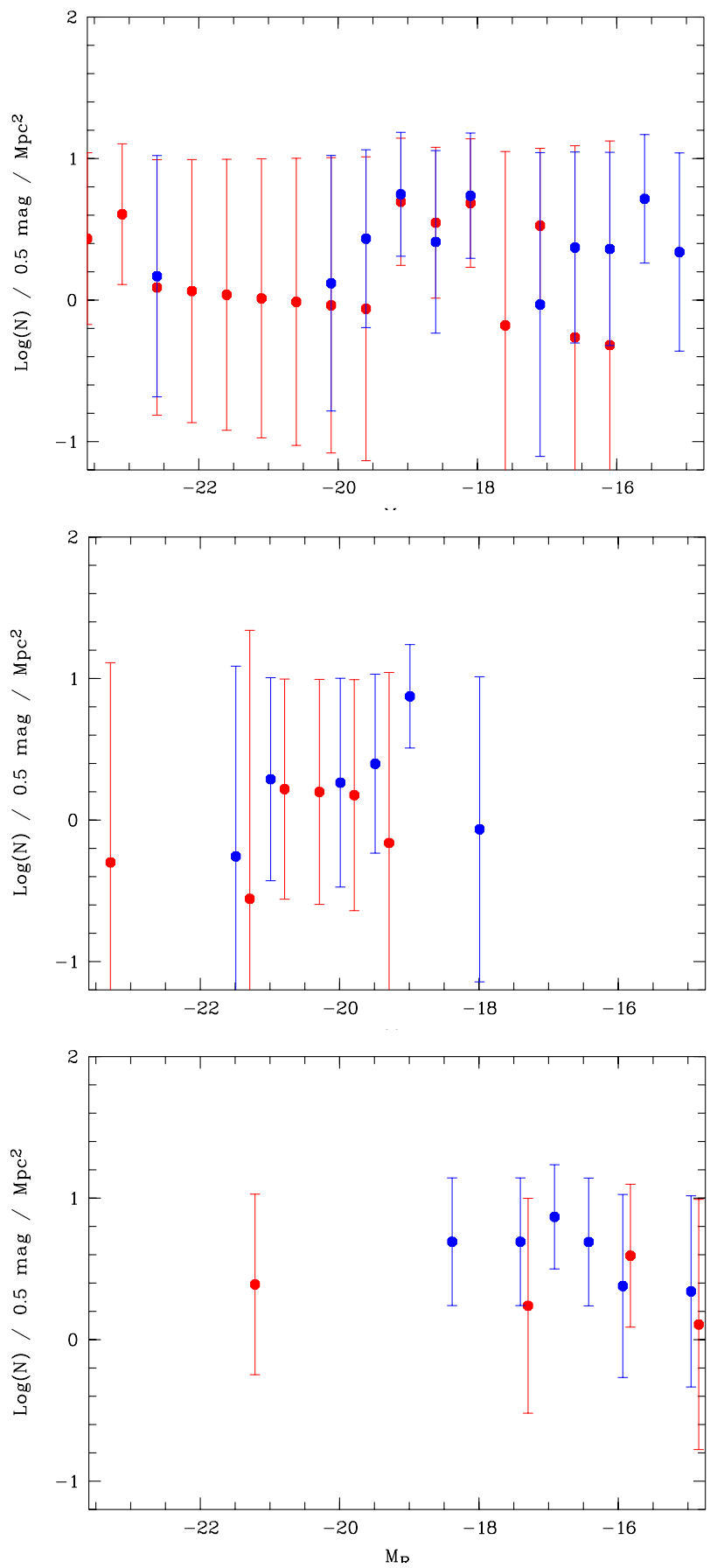

Fig. 16. Photometric redshift-based $R c$ band galaxy luminosity functions of NGC 6034 (top), 1RXS J235814.4+150524 (middle), and RX J1119.7+2126 (bottom). Red dots are for early-type or early spiral galaxies (types $\leq 34$ ) and blue dots are for late spiral galaxies (types $\geq 35$ ).

luminosity functions. For example, we showed that in massive structures such as the Coma cluster, the faint parts of the luminosity functions were mainly populated by late-type objects (see Adami et al. 2007a). We detect a similar behavior for the groups (see Fig. 16). Keeping in mind that we are dealing with catalogs of potential structure members biased toward early-type galaxies and despite the less efficient selection of group members for latetype galaxies, these galaxies are dominant compared to the early photometric types at the faintest magnitudes. 


\subsection{Galaxy stellar population ages}

We confirmed in Adami et al. (2008) that short bursts of induced star formation in galaxies that are just enter to the potential well of structures were likely to occur in Coma. Since fossil groups are expected to have typical X-ray gas contents, we should detect the same behavior. We therefore computed these ages for the spectroscopic group member galaxy samples. By fixing the redshift to the spectroscopic value, we minimized the number of free parameters in the BC03 template fitting. Despite the limited samples, Figs. 9-11 seem to show that the galaxies with recent stellar populations are preferentially located in the group outskirts.

For RX J1119.7+2126, there is one galaxy very close to the dominant galaxy that also shows a relatively young stellar population (4 Gyr versus $11 \mathrm{Gyr}$ for the dominant galaxy). This could be explained if this small galaxy already experienced close encounters with the dominant galaxy, which could have induced bursts of star formation. Another explanation would be that this galaxy is not dynamically linked to the group and is just a young group-passing-through object, although its spectroscopic redshift $(z=0.06134)$ is very similar to the group redshift $(z=0.061)$.

\section{Summary}

By gathering imaging and spectroscopic data from various sources, we have analysed the properties of three groups of galaxies. Our main results are the following:

- The large-scale environment of 1RXS J235814.4+150524 is quite poor and diffuse (even if its galaxy density map does show a clear signature of a surrounding cosmic web), and therefore cannot provide many infalling galaxies. RX J1119.7+2126 appears to be very isolated, as previously shown by Adami et al. (2007b). On the other hand, Lopes de Oliveira et al. (2010) have shown that the cosmic environment of NGC 6034 is very rich.

- At the group scale, 1RXS J235814.4+150524 shows no substructure and seems to have a flatter density profile in its center than NGC 6034. RX J1119.7+2126 do not show any galaxies present in the immediate vicinity of the dominant galaxy.

- A red sequence is discernable for all three groups in a color-magnitude diagram. The luminosity functions derived with photometric redshift selection and with statistical background subtraction show comparable shapes, and also agree with the few points obtained based on spectroscopic redshifts. The two fossil groups show a dip after the dominant galaxy. Luminosity functions have a regular shape down to the completeness level for RX J1119.7+2126 and NGC 6034 with a nearly flat slope for the faintest magnitudes. There is clear lack of faint galaxies for 1RXS J235814.4+150524 for the faintest magnitudes. This agrees well with the slightly decreasing luminosity functions of Proctor et al. (2011) at $M_{R} \geq-20$ when considering areas inside half the virial radius. The faint parts of the luminosity functions of our two fossil groups finally appear dominantly populated by latetype galaxies.

- Galaxies with recent stellar populations seem preferentially located in the group outskirts. This is expected if bursts of star formation occured when these galaxies entered the groups.

- RX J1119.7+2126 is definitely classified as a fossil group; 1RXS J235814.4+150524 also has properties very close to those of a fossil group, while we confirm that NGC 6034 is a normal group.

Acknowledgements. The authors thank the referee for useful and constructive comments. We are grateful to the organizers of the 6th Neon Observing School. We gratefully acknowledge the contributions of the students of the 2005/2008 classes of the Aix-Marseille I AER M2. We also thank the whole XMM-LSS team for their help. Based on observations made at Observatoire de Haute Provence (CNRS), France, at Asiago Observatory (Italy), at TNG (La Palma, Spain), and with ESO Telescopes at the La Silla Observatory under programme ID 082.A-0374. Also based on the use of the NASA/IPAC Extragalactic Database (NED) which is operated by the Jet Propulsion Laboratory, California Institute of Technology, under contract with the National Aeronautics and Space Administration, and on the use of the IA2-TNG archive (constructed as part of the activities of the Italian Theoretical Virtual Observatory). Funding for the SDSS and SDSS-II has been provided by the Alfred P. Sloan Foundation, the Participating Institutions, the National Science Foundation, the US Department of Energy, the National Aeronautics and Space Administration, the Japanese Monbukagakusho, the Max Planck Society, and the Higher Education Funding Council for England. The SDSS Web Site is http://www . sdss.org/. The SDSS is managed by the Astrophysical Research Consortium for the Participating Institutions. The Participating Institutions are the American Museum of Natural History, Astrophysical Institute Potsdam, University of Basel, University of Cambridge, Case Western Reserve University, University of Chicago, Drexel University, Fermilab, the Institute for Advanced Study, the Japan Participation Group, Johns Hopkins University, the Joint Institute for Nuclear Astrophysics, the Kavli Institute for Particle Astrophysics and Cosmology, the Korean Scientist Group, the Chinese Academy of Sciences (LAMOST), Los Alamos National Laboratory, the Max-Planck-Institute for Astronomy (MPIA), the Max-Planck-Institute for Astrophysics (MPA), New Mexico State University, Ohio State University, University of Pittsburgh, University of Portsmouth, Princeton University, the United States Naval Observatory, and the University of Washington.

\section{References}

Adami, C., Nichol, R. C., Mazure, A., et al. 1998, A\&A, 334, 765 Adami, C., Durret, F., Mazure, A., et al. 2007a, A\&A, 462, 411

Adami, C., Russeil, D., \& Durret, F. 2007b, A\&A, 467, 459

Adami, C., Ilbert, O., Pelló, R., et al. 2008, A\&A, 491, 681

Adami, C., Mazure, A., Pierre, M., et al. 2011, A\&A, 526, A18

Arnouts, S., Cristiani, S., Moscardini, L., et al. 1999, MNRAS, 310, 540

Bertin, E. 2006, ASPC, 351, 112

Bertin, E., \& Arnouts, S. 1996, A\&A, 117, 393

Bertin, E., Mellier, Y., \& Radovich, M. 2002, ASPC, 281, 228

Bruzual, G., \& Charlot, S. 2003, MNRAS, 344, 1000

Carlberg, R. G., Yee, H. K. C., Ellingson, E., et al. 1997, ApJ, 485, L13

Calzetti, D., \& Heckman, T. M. 1999, ApJ, 519, 27

Cousins, A. W. J. 1973, Mem RAS, 77, 223

Cousins, A. W. J. 1974, MNSSA, 33, 149

Cypriano, E. S., Mendes de Oliveira, C., \& Sodré, L. Jr. 2006, AJ, 132, 514

Dunkley, J., Komatsu, E., Nolta, M. R., et al. 2009, ApJS, 180, 306

Garilli, B., Fumana, M., Franzetti, P., et al. 2010, PASP, 122, 827

Guennou, L., Adami, C., Ulmer, C., et al. 2010, A\&A, 523, A21

Hoyle, F., \& Vogeley, M. S. 2004, ApJ, 607, 751

Ilbert, O., Tresse, L., Zucca, E., et al. 2005, A\&A, 439, 863

Ilbert, O., Arnouts, S., McCracken, H. J., et al. 2006, A\&A, 457, 841

Jones, L. R., Ponman, T. J., Horton, A., et al. 2003, MNRAS, 343, 627

Kodama, T., \& Arimoto, N. 1997, A\&A, 320, 41

Lopes de Oliveira, R., Carrasco, E. R., Mendes de Oliveira, C., et al. 2010, AJ, 139,216

Mulchaey, J. S., \& Zabludoff, A. I. 1999, ApJ, 514, 133

Proctor, R. N., Mendes de Oliveira, C., Dupke, R., et al. 2011, MNRAS, 418, 2054

Santos, W. A., Mendes de Oliveira, C., \& Sodré, L. Jr. 2007, AJ, 134, 1551

Schneider, D. P., Gunn, J. E., \& Hoessel, J. G. 1983, ApJ, 264, 337

Serna, A., \& Gerbal, D. 1996, A\&A, 309, 65

Thuan, T. X., \& Gunn, J. E. 1976, PASP, 88, 543

Ulmer, M. P., Adami, C., Covone, G., et al. 2005, ApJ, 624, 124

Yoshioka, T., Furuzawa, A., Takahashi, S., et al. 2004, Adv. Space Res., 34, 2525

Pages 13 to 14 are available in the electronic edition of the journal at http://www . aanda.org 


\section{Appendix A: Spectroscopic redshifts}

This section gives the measured spectroscopic redshifts for the three groups (Tables A.1-A.4) and a typical spectrum for the bright galaxies of the wide study of the neighborhood of 1RXS J235814.4+150524 (2MASX J23480439+1442557: Fig. A.1).

Table A.1. Redshifts used for the deep study of the central area of RX J1119.7+2126.

\begin{tabular}{|c|c|c|c|c|}
\hline RA & Dec & $z$ & Nature & Source \\
\hline 169.86131 & 21.43701 & 0.362 & galaxy & TNG \\
\hline 169.87387 & 21.44891 & $0.261 / 0.261$ & galaxy & TNG/TNG \\
\hline 169.88421 & 21.46373 & 0.2595 & galaxy & TNG \\
\hline 169.88604 & 21.38743 & 1.422 & active & TNG \\
\hline 169.88833 & 21.46607 & 0.28100 & galaxy & NED \\
\hline 169.89172 & 21.40683 & 0.3379 & galaxy & TNG \\
\hline 169.90656 & 21.43067 & 0. & star & TNG \\
\hline 169.90868 & 21.44097 & 2.97 & active & TNG \\
\hline 169.90989 & 21.39666 & 0.3065 & galaxy & TNG \\
\hline 169.91870 & 21.43216 & 0.2755 & galaxy & TNG \\
\hline 169.92206 & 21.45909 & 0.361 & galaxy & TNG \\
\hline 169.92260 & 21.46334 & 0. & star & TNG \\
\hline 169.92296 & 21.42640 & 0.4345 & galaxy & TNG \\
\hline 169.92513 & 21.43622 & $0.06134 *$ & galaxy & NED \\
\hline 169.92680 & 21.45457 & 0.392 & galaxy & TNG \\
\hline 169.93159 & 21.44756 & $0.06055 / 0.0603 / 0.0603 *$ & galaxy & NED/TNG/TNG \\
\hline 169.93549 & 21.46902 & 0.306 & galaxy & TNG \\
\hline 169.93623 & 21.53648 & 0.14058 & galaxy & NED \\
\hline 169.93633 & 21.51504 & 0.2275 & galaxy & TNG \\
\hline 169.93824 & 21.51931 & $0.22931 / 0.228$ & galaxy & NED/TNG \\
\hline 169.94032 & 21.50432 & 0.153 & galaxy & TNG \\
\hline 169.94221 & 21.42251 & 0.3975 & galaxy & TNG \\
\hline 169.94622 & 21.48221 & $0.21409 / 0.2125 / 0.213$ & galaxy & NED/TNG/TNG \\
\hline 169.96226 & 21.51082 & $0.0565 * ?$ & galaxy & TNG \\
\hline 169.96593 & 21.51904 & 0.405 & galaxy & TNG \\
\hline 169.96682 & 21.50062 & $0.08247 / 0.082$ & galaxy & NED/TNG \\
\hline 169.96832 & 21.41800 & 0.1652 & galaxy & TNG \\
\hline 169.98881 & 21.44085 & $0.059 *$ & galaxy & TNG \\
\hline 169.99585 & 21.53157 & $0.05976 *$ & galaxy & NED \\
\hline 169.99891 & 21.45859 & 0.299 & galaxy & TNG \\
\hline 170.00296 & 21.44578 & $0.2095 / 0.2104$ & galaxy & TNG/TNG \\
\hline 170.00600 & 21.43058 & 0.429 & galaxy & TNG \\
\hline
\end{tabular}

Notes. Columns 1 and 2: J2000.0 coordinates of the object (in decimal degrees); Col. 3: redshift (the number of significant digits depends on the spectrum quality, galaxies marked with a* are potential group members); Col. 4: nature of the object (galaxy, active object, star); Col. 5: source (TNG, NED). The typical uncertainty on non-literature redshifts is 0.001 . Given the low signal-to-noise ratio of the TNG spectra, we chose to use mainly literature redshifts when available. We note that the group velocity dispersion was computed without the $z=0.0565$ galaxy.

Table A.2. Redshifts used for the wide study of the neighborhood of 1RXS J235814.4+150524.

\begin{tabular}{lcccc}
\hline \hline Name & $z$ & $r^{\prime}$ & Ins. & $\begin{array}{c}\text { Exp. time } \\
(\mathrm{h})\end{array}$ \\
\hline 2MASX J23475868+1410367 & 0.0709 & 15.92 & Carelec & 1 \\
2MASX J23480439+1442557 & 0.1052 & 16.16 & B \&C & 1 \\
SDSS J234826.30+160113.7 & 0.0012 & 14.17 & Carelec & 1 \\
2MASX J23510162+1527106 & 0.2510 & 15.49 & AFOSC & 1 \\
2MASX J23530977+1503278 & 0.0786 & 15.53 & B \&C & 2.5 \\
2MASX J23543897+1604501 & 0.0755 & 16.06 & B \&C & 2.5 \\
2MASX J23543994+1604561 & 0.0885 & 15.12 & B \&C & 2.5 \\
2MASX J23551805+1531545 & 0.0747 & 15.98 & Carelec & 1 \\
KUG 2358+159 & 0.0440 & 15.87 & Carelec & 1 \\
2MASX J23582694+1513586 & 0.1527 & 16.04 & AFOSC & 2 \\
2MASX J23592068+1440129 & 0.0928 & 16.06 & AFOSC & 2 \\
2MASX J00001187+1405240 & 0.1035 & 15.96 & Carelec & 1 \\
2MASX J00040323+1420083 & 0.0383 & 15.67 & Carelec & 1 \\
SDSS J000547.13+160838.1 & 0.1163 & 16.02 & Carelec & 1 \\
2MASX J00061715+1354269 & 0.0757 & 16.18 & AFOSC & 0.5 \\
2MASX J00075853+1510533 & 0.1131 & 16.00 & Carelec & 1 \\
\hline
\end{tabular}

Notes. Column 1: NED galaxy name; Col. 2: redshift; Col. 3: SDSS $r^{\prime}$ magnitude; Col. 4: instrument (Carelec, Boller \& Chivens or AFOSC); Col. 5: exposure time. The typical redshift uncertainty for these galaxies is 0.0005 and the spectral resolution is $7 \AA / p x$. 
Table A.3. Redshifts used for the deep study of the central area of 1RXS J235814.4+150524.

\begin{tabular}{lccc}
\hline \hline RA & Dec & $z$ & Source \\
\hline 359.49886 & 15.10602 & 0.1527 & TNG \\
359.50628 & 15.11972 & $0.1778 *$ & TNG \\
359.51304 & 15.11320 & $0.1786 *$ & TNG \\
359.51911 & 15.14732 & $0.1783 *$ & TNG \\
359.52384 & 15.14407 & 0.1553 & TNG \\
359.53523 & 15.12241 & $0.1471 / 0.15539$ & TNG/NED \\
359.54073 & 15.11647 & 0.0455 & TNG \\
359.54074 & 15.09299 & $0.1804 *$ & TNG \\
359.54119 & 15.05579 & $0.0951 / 0.09537$ & TNG/NED \\
359.54428 & 15.07865 & 0.1748 & TNG \\
359.54479 & 15.10169 & 0.175 & TNG \\
359.55371 & 15.06013 & $0.1775 / 0.18900 *$ & TNG/NTT \\
359.55694 & 15.09995 & $0.1795 *$ & TNG \\
359.55717 & 15.10495 & 0.1288 & TNG \\
359.55917 & 15.11952 & 0.4105 & TNG \\
359.55965 & 15.14777 & 0.1895 & TNG \\
359.56001 & 15.09075 & 0.1769 & TNG \\
359.56027 & 15.15464 & 0.1531 & TNG \\
359.56263 & 15.04156 & $0.1783 *$ & TNG \\
359.56280 & 15.09560 & $0.1786 / 0.1781 *$ & TNG/NED \\
359.56301 & 15.09571 & $0.1781 / 0.17843 *$ & TNG/NED \\
359.56370 & 15.04343 & 0.1902 & TNG \\
359.56425 & 15.06989 & 0.3460 & TNG \\
359.56460 & 15.06430 & $0.1547 / 0.1535$ & TNG/NED \\
359.56483 & 15.06406 & 0.1535 & TNG \\
359.56519 & 15.08331 & 0.1891 & TNG \\
359.56843 & 15.13146 & $0.4937 / 0.4460$ & TNG/NED \\
359.56862 & 15.13926 & 0.1590 & TNG \\
359.56865 & 15.13907 & 0.1549 & TNG \\
359.56870 & 15.13147 & 0.446 & TNG \\
359.56910 & 15.09212 & $0.1794 *$ & TNG \\
359.56978 & 15.05974 & $0.1783 *$ & TNG \\
359.57040 & 15.10639 & 0.1755 & TNG \\
359.57131 & 15.09885 & $0.1745 / 0.1793$ & TNG/NTT \\
359.57407 & 15.15178 & 0.2961 & TNG \\
359.57923 & 15.08278 & 0.4938 & TNG \\
359.58374 & 15.10016 & $0.1783 *$ & TNG \\
359.58437 & 15.13468 & $0.1785 *$ & TNG \\
359.59139 & 15.10234 & 0.1824 & TNG \\
359.59736 & 15.11437 & $0.179 *$ & TNG \\
359.60624 & 15.06930 & 0.1520 & TNG \\
359.61164 & 15.06756 & 0.1865 & TNG \\
359.62673 & 15.07429 & 0.1675 & TNG \\
\hline & & &
\end{tabular}

Notes. Columns 1 and 2: coordinates of the object (decimal degrees); Col. 3: redshift (the number of significant digits depends on the precision achieved for the redshift determination), galaxies marked with a $*$ are potential group members); Col. 4: source (TNG, NTT or NED). The typical redshift uncertainty of the non-literature redshifts is 0.001 . Given the good quality of the TNG spectra, we chose to use mainly these spectra.
Table A.4. Redshifts used for the deep study of the central area of NGC 6034, taken from the literature (SDSS).

\begin{tabular}{lcc}
\hline \hline RA & Dec & $z$ \\
\hline 240.75500 & 17.26940 & 0.111570 \\
240.76401 & 17.24150 & 0.134560 \\
240.76801 & 17.19090 & $0.036130 *$ \\
240.76801 & 17.24870 & 0.135860 \\
240.77200 & 17.19340 & $0.033120 *$ \\
240.77400 & 17.17230 & $0.033590 *$ \\
240.81200 & 17.23930 & $0.035130 *$ \\
240.82401 & 17.18380 & $0.034860 *$ \\
240.84399 & 17.05720 & 0.038910 \\
240.85300 & 17.28030 & 0.134290 \\
240.86600 & 17.27470 & 0.135400 \\
240.86700 & 17.19620 & $0.034520 *$ \\
240.86700 & 17.27220 & 0.134850 \\
240.87100 & 17.15840 & $0.035360 *$ \\
240.87900 & 17.18240 & $0.033770 *$ \\
240.88400 & 17.19840 & $0.033880 *$ \\
240.88800 & 17.16310 & $0.033180 *$ \\
240.89600 & 17.25580 & $0.034770 *$ \\
240.91400 & 17.18480 & $0.034080 *$ \\
240.91901 & 17.33800 & 0.042880 \\
240.92000 & 17.06900 & 0.137350 \\
240.92900 & 17.30370 & 0.096960 \\
240.93401 & 17.29740 & $0.035570 *$ \\
240.93600 & 17.28870 & 0.097110 \\
240.93700 & 17.13570 & 0.037940 \\
240.93700 & 17.23070 & $0.033110 *$ \\
240.95100 & 17.24060 & $0.036530 *$ \\
240.96100 & 17.23950 & 0.042600 \\
240.97701 & 17.07650 & $0.034850 *$ \\
240.98500 & 17.11100 & $0.034780 *$ \\
240.98599 & 17.30510 & $0.033390 *$ \\
240.99001 & 17.23890 & 0.139580 \\
241.00600 & 17.13920 & 0.099240 \\
241.01100 & 17.28240 & $0.033470 *$ \\
241.02299 & 17.22080 & 0.295020 \\
241.03000 & 17.23640 & 0.031450 \\
241.03200 & 17.20700 & $0.035030 *$ \\
241.04300 & 17.20510 & $0.034840 *$ \\
\hline & & \\
& & \\
& $*$
\end{tabular}

Notes. Columns 1 and 2: coordinates of the object (decimal degrees); Col. 3: redshift, galaxies marked with a $*$ are potential group members).

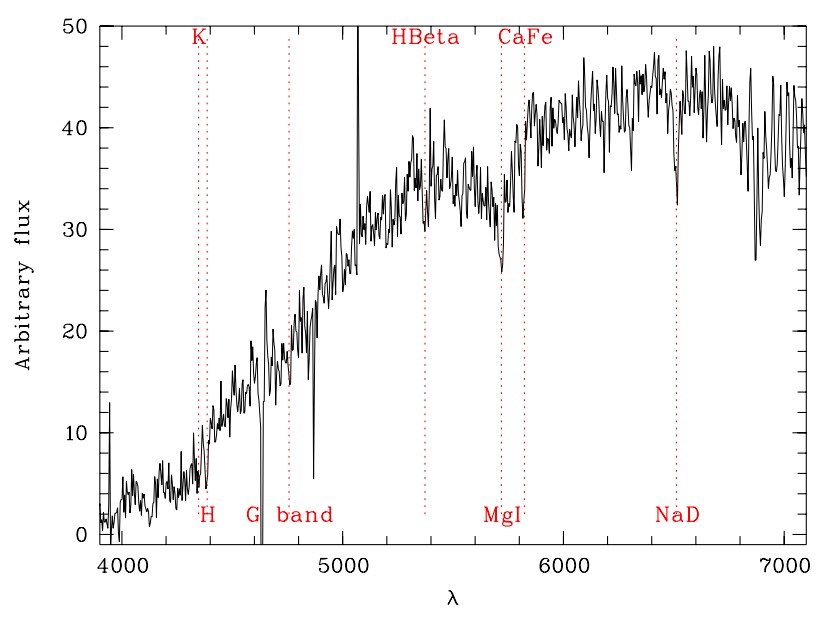

Fig. A.1. Spectrum of 2MASX J23480439+1442557 observed with the B \&C spectrograph. The identified lines are shown in red. 